ACCEPTED MANUSCRIPT

\title{
Hydrogel co-networks of gelatine methacrylate and poly(ethylene glycol) diacrylate sustain 3D functional $<i>$ in vitro $</ i>$ models of intestinal mucosa
}

To cite this article before publication: Anna Vila et al 2019 Biofabrication in press https://doi.org/10.1088/1758-5090/ab5f50

\section{Manuscript version: Accepted Manuscript}

Accepted Manuscript is "the version of the article accepted for publication including all changes made as a result of the peer review process, and which may also include the addition to the article by IOP Publishing of a header, an article ID, a cover sheet and/or an 'Accepted

Manuscript' watermark, but excluding any other editing, typesetting or other changes made by IOP Publishing and/or its licensors"

This Accepted Manuscript is @ 2019 IOP Publishing Ltd.

During the embargo period (the 12 month period from the publication of the Version of Record of this article), the Accepted Manuscript is fully protected by copyright and cannot be reused or reposted elsewhere.

As the Version of Record of this article is going to be / has been published on a subscription basis, this Accepted Manuscript is available for reuse under a CC BY-NC-ND 3.0 licence after the 12 month embargo period.

After the embargo period, everyone is permitted to use copy and redistribute this article for non-commercial purposes only, provided that they adhere to all the terms of the licence https://creativecommons.org/licences/by-nc-nd/3.0

Although reasonable endeavours have been taken to obtain all necessary permissions from third parties to include their copyrighted content within this article, their full citation and copyright line may not be present in this Accepted Manuscript version. Before using any content from this article, please refer to the Version of Record on IOPscience once published for full citation and copyright details, as permissions will likely be required. All third party content is fully copyright protected, unless specifically stated otherwise in the figure caption in the Version of Record.

View the article online for updates and enhancements. 


\title{
Hydrogel co-networks of gelatine methacrylate and poly(ethylene glycol) diacrylate sustain 3D functional in vitro models of intestinal mucosa
}

\author{
Anna Vila ${ }^{1, \#}$, Núria Torras ${ }^{1, \#}$, Albert G. Castaño ${ }^{1}$, María García-Díaz ${ }^{1}$, Jordi \\ Comelles $^{1}$, Teresa Pérez-Berezo ${ }^{1}$, Carmen Corregidor ${ }^{1}$, Óscar Castaño ${ }^{3,1,2}$, Elisabeth \\ Engel $^{1,2,4}$, Vanesa Fernández-Majada ${ }^{1, *}$ and Elena Martínez ${ }^{1,2,3, *}$
}

${ }^{1}$ Institute for Bioengineering of Catalonia (IBEC), The Barcelona Institute of Science and Technology (BIST), Barcelona, Spain ${ }^{2}$ Centro de Investigación Biomédica en Red (CIBER), Madrid, Spain

${ }^{3}$ Electronics and Biomedical Engineering, University of Barcelona (UB), Barcelona, Spain

${ }^{4}$ Materials Science and Metallurgical Engineering, Polytechnical University of Catalonia (UPC), Barcelona, Spain

\#equal contribution

*Corresponding authors: emartinez@ibecbarcelona.eu, vfernandez@ibecbarcelona.eu

Received xxxxxx

Accepted for publication $\mathrm{xxxxxx}$

Published xxxxxx

\begin{abstract}
Mounting evidence supports the importance of the intestinal epithelial barrier and its permeability both in physiological and pathological conditions. Conventional in vitro models to evaluate intestinal permeability rely on the formation of tightly packed epithelial monolayers grown on hard substrates. These two-dimensional (2D) models lack the cellular and mechanical components of the non-epithelial compartment of the intestinal barrier, the stroma, which are key contributors to the barrier permeability in vivo. Thus, advanced in vitro models approaching the in vivo tissue composition are fundamental to improve precision in drug absorption predictions, to provide a better understanding of the intestinal biology, and to faithfully represent related diseases. Here, we generate photo-crosslinked gelatine methacrylate (GelMA) - poly(ethylene glycol) diacrylate (PEGDA) hydrogel co-networks that provide the required mechanical and biochemical features to mimic both the epithelial and stromal compartments of the intestinal mucosa, i.e., they are soft, cell adhesive and cell-loading friendly, and suitable for long-term culturing. We show that fibroblasts can be embedded in the GelMA-PEGDA hydrogels while epithelial cells can grow on top to form a mature epithelial monolayer that exhibits barrier properties which closely mimic those of the intestinal barrier in vivo, as shown by the physiologically relevant transepithelial electrical resistance (TEER) and permeability values. The presence of fibroblasts in the artificial stroma compartment accelerates the formation of the epithelial monolayer and boosts the recovery of the epithelial integrity upon temporary barrier disruption, demonstrating that our system is capable of successfully reproducing the interaction between different cellular compartments. As such, our hydrogel co-networks offer a technologically simple yet sophisticated approach to produce functional three-dimensional (3D) in vitro models of epithelial barriers with epithelial and stromal cells arranged in a spatially relevant manner and near-physiological functionality.
\end{abstract}

Keywords: Gelatine methacrylate, poly(ethylene glycol) diacrylate, GelMA-PEGDA co-networks, intestinal mucosa, epithelial-stromal interactions, intestinal barrier function, intestinal permeability, hydrogels

\section{Introduction}

The intestinal epithelium consists of a layer of tightly packed interconnected epithelial cells sitting on a collagen- based basal lamina that separates it from the stromal tissue (named lamina propria) below. The intestinal lamina propria contains many cell types including fibroblasts, myofibroblasts, endothelial and immune cells embedded in a matrix [1]. The intestinal epithelium in combination with the 
lamina propria form the intestinal mucosa, which acts as a pseudo-permeable barrier and ensures adequate containment of undesirable luminal contents while preserving the ability to absorb nutrients and beneficial substances [2]. It has been suggested that these barrier and permeability functions of the intestinal mucosa could play a crucial role in both physiological and pathological conditions [3]. For instance, intestinal drug permeability is a key parameter to take into account in the early stages of drug discovery $[4,5]$. At the same time, intestinal barrier dysfunction leading to an increased intestinal permeability has been associated to numerous health conditions such as inflammatory bowel diseases, irritable bowel syndrome, celiac disease, obesity, and metabolic diseases [6]. Whether the inflammation associated with these diseases is a cause or a consequence of the increased intestinal permeability is still not fully understood [7]. Traditionally, intestinal permeability studies have been performed in vivo or using animal mucosal tissue explants. Although valuable, these experiments provide few mechanistic insights due to the complexity of the in vivo environment [8]. Conversely, in vitro models, which mostly use transformed epithelial cell lines grown as monolayers, provide a limited and simplistic representation of only the epithelial component of the intestinal mucosa. However, it has been demonstrated that epithelium-stroma interactions are important to maintain the intestinal mucosa integrity [9]. Thus, the development of more physiologically relevant $3 \mathrm{D}$ intestinal mucosa-like in vitro models that represent the epithelial and the stromal compartments of the tissue in a spatially-relevant manner would be instrumental to reduce the gap between in vitro epithelial cell-based models and in vivo experiments.

Engineered tissues represent a new paradigm in the field of cell-based in vitro assays. They combine biofabrication and tissue-engineering components to provide in vitro models with tissue-like characteristics such as 3D architecture, multicellularity, cell-matrix interactions and near-in vivo functionality [10-13]. As such, engineered tissues hold the potential to deliver huge improvements over current reductionist cell culture monolayers, opening new research avenues by providing better tools for basic research, disease modelling, and drug testing. However, examples of engineered tissues recreating the intestinal mucosa and, more specifically, including stromal and epithelial components, are scarce. In a pioneering work, collagen hydrogels were used to embed primary mouse fibroblasts and produce a 3D model of the intestinal mucosa that included lamina propria and epithelial cells [14]. This models yielded improved predictions on drug permeability with respect to standard in vitro cultures based on tightly packed Caco-2 cell monolayers on Transwell ${ }^{\circledR}$, which typically show abnormally high TEER values and low permeability values [14]. However, this model used fibroblasts with a low passage number to represent the stromal component, which compromises its adoption in routine long-term applications. A different study employed fibronectin-gelatine nanofilms to produce 3D-multilayer structures consisting of normal dermal fibroblasts underneath a monolayer of epithelial Caco-2 cells [15]. The authors showed that the presence of multiple layers of dermal fibroblasts accelerates Caco-2 epithelial monolayer formation and enhances epithelial barrier function. In contrast, a more recent study showed how CCD-18Co intestinal fibroblasts embedded in Matrigel ${ }^{\mathbb{}}$ were able to sustain the growth of epithelial cells, sitting on top of them, through the production of extracellular matrix proteins. The CCD-18Co fibroblasts interfered with barrier robustness by getting intercalated between the epithelial cells [16]. The discrepancies reported with respect to the effects of stromal fibroblasts on the epithelial barrier performance is likely due to differences in experimental set-ups, particularly regarding the origin and density of the fibroblasts, the thickness of the construct, and the 3D organization of the epithelial and stromal compartments. As the literature in the field is sparse, further analysis of the impact of fibroblasts on the maintenance of the epithelial barrier maintenance is needed to elucidate the underlying mechanisms.

Thus far, most approaches to engineer 3D in vitro models of the intestinal mucosa that represent both the epithelial and stromal compartments, have employed hydrogels of natural origin as extracellular matrices (ECM). Typically, hydrogels are the preferred choice because they possess a high water content, mechanical properties that are similar to those in the native ECM of soft tissues, and confer diffusion-driven solute transport properties $[17,18]$. A major drawback of natural hydrogels is that they are highly biodegradable. On the one hand, this biodegradability allows for cell matrix remodelling, which can have positive effects on cell proliferation and migration $[19,20]$. On the other hand, it limits the available time for cell culturing and compromises the mechanical stability of the engineered tissues. Often, this problem is overcome by increasing the density of cells on the scaffold. However, this typically results in a density of cells that exceeds physiologically realistic levels in the native lamina propria and thus further removes these models from being able to faithfully represent in vivo conditions [21].

In this work, we report a simple strategy to engineer a 3D model of the small intestinal mucosa that includes both the epithelium and the stroma components. With this aim, we employ hydrogels based on the co-polymerization of gelatine methacrylate (GelMA) and poly(ethylene glycol) diacrylate (PEGDA) polymers to produce cell-laden constructs. GelMA provides cell-adhesive and biodegradable moieties, but its mechanical robustness is insufficient to sustain long-term cultures [17]. Conversely, PEGDA is a synthetic, nondegradable polymer that forms mechanically stable networks. We demonstrate that GelMA-PEGDA hydrogel co-networks generated by photopolymerization possess tuneable 
physicochemical and mechanical properties depending on their polymer composition and total macromer content. For a particular co-network composition, we proved that fibroblasts-laden hydrogels support the formation of a mature epithelial monolayer, providing an engineered in vitro model of the epithelial and stromal compartments. We then demonstrate that while our approach is sufficiently simple to be used with conventional cell culture systems, it also possesses the necessary complexity to recapitulate the relevant epithelial-stromal interactions, yielding realistic TEER values and an improved representation of the permeability of drug model compounds. The model can therefore be used to improve the predictive accuracy of state-of-the-art in vitro systems.

\section{Materials and Methods}

\subsection{GelMA synthesis and characterization}

Gelatine methacrylate (GelMA) was prepared following the method described previously [22,23]. Briefly, a 10\% (w/v) gelatine solution was obtained by dissolving gelatine from porcine skin type A (Sigma-Aldrich) in phosphate buffer saline (PBS; pH 7.4) (Gibco) at $50^{\circ} \mathrm{C}$ under stirring conditions for approximately $2 \mathrm{~h}$. Methacrylic anhydride (MA) (SigmaAldrich) at $5 \% \mathrm{v} / \mathrm{v}$ was added at a rate of $0.5 \mathrm{~mL} \cdot \mathrm{min}^{-1}$ and left to react for 1 hour while stirring. Then, the solution was centrifuged at $1200 \mathrm{rpm}$ for $3 \mathrm{~min}$ and the reaction was stopped by adding Milli-Q water to the supernatant. The resulting solution was dialyzed using 6-8 $\mathrm{kDa}$ of molecular weight cut-off (MWCO) membranes (Spectra/por, Spectrumlabs) against Milli-Q water at $40^{\circ} \mathrm{C}$, which was replaced every $4 \mathrm{~h}$ for 3 days. The $\mathrm{pH}$ of the dialyzed products was adjusted to 7.4. Samples were frozen overnight at $-80^{\circ} \mathrm{C}$ and lyophilized for 4-5 days (Freeze Dryer Alpha 1-4 LD Christ). The resulting products were stored at $-20^{\circ} \mathrm{C}$ until further use.

The successful methacrylation of the synthesized GelMA was analysed by nuclear magnetic resonance (NMR) spectroscopy [24]. ${ }^{1} \mathrm{H}-\mathrm{NMR}$ spectra were acquired using a Bruker DMX-500 high resolution NMR spectrometer (400 $\mathrm{MHz}$ ) (Bruker). Spectra were collected from nonmethacrylate gelatine as a control. To that end, GelMA solutions $(1 \mathrm{~mL})$ were prepared at $10 \mathrm{mg} \cdot \mathrm{mL}^{-1}$ in deuterium oxide. All spectra were collected with Mnova NMR software (Mestrelab Research). To quantitatively determine the amount of methacrylate amines in the GelMA polymer solutions, we employed the Habeeb Method [25]. This method is based on the fact that primary amines react with 2,4,6-Trinitrobenzene sulfonic acid (TNBS) (Sigma-Aldrich) to form a highly chromogenic derivative solution that can be measured by absorbance. Briefly, gelatine and GelMA polymers were dissolved at a concentration of $0.5 \mathrm{mg} \cdot \mathrm{mL}^{-1}$ in sodium bicarbonate buffer (NaHCO3, 0.1M; pH 8.4, in Milli-Q water)
(Sigma-Aldrich) at $40^{\circ} \mathrm{C}$ under stirring conditions. To generate a standard curve, $100 \mu \mathrm{L}$ of increasing concentrations of gelatine solution and $100 \mu \mathrm{L}$ of GelMA solution at 0.5 $\mathrm{mg} \cdot \mathrm{mL}^{-1}$ were placed in a 96 well plate. $50 \mu \mathrm{L}$ of TNBS $0.01 \%$ solution in sodium bicarbonate buffer was added to each well and the mixture was incubated for $2 \mathrm{~h}$ at $37^{\circ} \mathrm{C}$ in complete darkness. The TNBS reaction was stopped by adding $50 \mu \mathrm{L}$ of sodium dodecyl sulfate 10\% (SDS) (Sigma-Aldrich) and 25 $\mu \mathrm{L}$ of hydrochloric acid ( $\mathrm{HCl}, 1 \mathrm{M})$ (Panreac) to each well. The absorbance of the resulting solutions was measured at 335 $\mathrm{nm}$ using a spectrophotometer (Infinite M200 PRO Multimode Microplate Reader, Tecan). A linear calibration curve that relates the absorbances of the gelatine solution titrations to the free amine percentage $(\mathrm{X})$ in the sample was established. Then, the degree of methacrylation of the GelMA samples was calculated as: methacrylated amines $(\%)=100$ $\mathrm{X}$.

\subsection{Fabrication of GelMA and GelMA-PEGDA hydrogels}

GelMA polymer solutions containing two different concentrations of total macromers $(7.5 \%$ and $12.5 \% \mathrm{w} / \mathrm{v})$ were dissolved in Dulbecco's modified eagle medium (DMEM) without Phenol red (Gibco, Thermo Fisher Scientific) and complemented with $1 \%$ Penicillin-Streptomycin (SigmaAldrich) at $65^{\circ} \mathrm{C}$ under stirring conditions. In addition, GelMA and PEGDA polymer solutions containing the same concentrations of total macromers $(7.5 \%$ and $12.5 \% \mathrm{w} / \mathrm{v})$ but different percentages of both polymers, namely $3.75 \% \mathrm{w} / \mathrm{v}$ GelMA- 3.75\% w/v PEGDA and 7.5\% w/v GelMA-5\% w/v PEGDA, were prepared as follows: PEGDA, with a molecular weight of $4000 \mathrm{Da}$ (Polysciences), was dissolved at $65^{\circ} \mathrm{C}$ in DMEM without Phenol red (Gibco, Thermo Fisher Scientific) and complemented with 1\% Penicillin-Streptomycin (SigmaAldrich) under stirring conditions for $2 \mathrm{~h}$. Then, PEGDA solutions were filtered and mixed with the corresponding percentages of GelMA. Next, 2-Hydroxy-4'-(2hydroxyethoxy)-2-methylpropiophenone photoinitiator (Irgacure D-2959) (Sigma-Aldrich) was added to the polymer solutions at a concentration of $0.5 \% \mathrm{w} / \mathrm{v}$ (see schemes in Figure 1(a)).

All polymer solutions were kept at $37^{\circ} \mathrm{C}$ for about $\sim 30 \mathrm{~min}$ before use. Hydrogel polymerization was performed following a previously described methodology [26]. Briefly, an array of circular pools (6.5 or $10 \mathrm{~mm}$ in diameter) were produced by punching polydimethylsiloxane (PDMS) Sylgard 184 (Dow Corning) membranes (prepared at a ratio 10:1 w/w between the prepolymer and the curing agent and cured at room temperature for $24 \mathrm{~h}$ ). The thickness of the hydrogel was defined by the height of the PDMS pools. Pools were mounted on top of polystyrene (PS) supports. Either silanized glass coverslips with a diameter of $12 \mathrm{~mm}$ or Tracketch ${ }^{\circledR}$ polyethylene terephthalate (PET) membranes with $5 \mu \mathrm{m}$ pore size (Sabeu $\mathrm{GmbH} \& \mathrm{Co}$ ) were then used as substrates 
covering the tops of the PDMS pools. Polymer solutions were poured into the pool array and flood exposed to UV light to form disc-shaped hydrogels. UV light exposure took place in a MJBA mask aligner (SUSS MicroTech) equipped with an iline filter $(\lambda=365 \mathrm{~nm})$ emitting an energy dose (power density $\mathrm{x}$ time) of $1.88 \mathrm{~J} \cdot \mathrm{cm}^{-2}$ (see scheme of the process in Figure 1(b)). Before each exposure, the incidental power density (power per surface unit) on the surface of the samples was measured using a UV-power meter (Model 1000, SUSS MicroTech) to properly adjust the exposure time. After UV exposure, unreacted polymer and photoinitiator were washed out with warmed $\mathrm{PBS}$ at $37^{\circ} \mathrm{C}$. Then, samples were kept submerged in $\mathrm{PBS}$ at $4^{\circ} \mathrm{C}$ to reach the equilibrium swelling before further characterization.

\subsection{Characterization of GelMA and GelMA-PEGDA hydrogels}

\subsubsection{Analysis of co-network homogeneity. We} determined the presence of GelMA and the microscopic homogeneity of the GelMA-PEGDA co-networks by the fluorescent labelling of the GelMA chains. For this purpose, discs of $10 \mathrm{~mm}$ in diameter and $1 \mathrm{~mm}$ in thickness, obtained by exposing to a UV dose of $3.00 \mathrm{~J} \cdot \mathrm{cm}^{-2}$, were fabricated onto glass coverslip substrates. Hydrogels were incubated overnight with a $0.02 \mathrm{mM}$ solution of Rhodamine-NHS (Sigma-Aldrich) at $4^{\circ} \mathrm{C}$, which labels GelMA chains. After extensive washing in PBS, hydrogels were imaged using confocal microscopy (LSM 800, Zeiss). The average intensity was measured from the maximum $\mathrm{Z}$ projections of stacks of images collected from the first $50 \mu \mathrm{m}$ of each hydrogel.

2.3.2 Mass swelling analysis. Hydrogel swelling was investigated on hydrogel discs $(10 \mathrm{~mm}$ in diameter and $1 \mathrm{~mm}$ in height) photopolymerized with a UV dose of $1.88 \mathrm{~J} \cdot \mathrm{cm}^{-2}$ onto glass coverslips covered with porous PET membranes ( 5 $\mu \mathrm{m}$ pore size). Since PET membranes tend to absorb UV light behaving as filters [26], they were used to fabricate hydrogels with mechanical properties comparable to those employed in the cell culture experiments. Right after polymerization, hydrogels were weighed $\left(\mathrm{m}_{\mathrm{c}}\right)$ and kept submerged in PBS at $37^{\circ} \mathrm{C}$ to induce swelling for one week, exchanging the PBS buffer every other day. Then, hydrogels were carefully wiped with a KimWipe tissue (Kimtech Science) to remove any excess liquid and weighed again $\left(\mathrm{m}_{\mathrm{s}}\right)$. Finally, samples were detached from the glass coverslips, frozen overnight at $-80^{\circ} \mathrm{C}$, lyophilized (Freeze Dryer Alpha 1-4 LD Christ) and weighed once more $\left(\mathrm{m}_{\mathrm{d}}\right)$. The mass swelling ratio for the GelMA and GelMA-PEGDA hydrogel co-networks was then calculated as the ratio between the mass due to swelling (difference between swollen and dry polymer) and the mass of the dry polymer.

$$
\text { Mass swelling ratio }=\left(\frac{m_{s}-m_{d}}{m_{d}}\right)
$$

\subsubsection{Diffusion coefficient and mesh size analysis.}

Molecular diffusion is an important network property of the hydrogels affecting mass transport. To estimate the molecular diffusion in the GelMA samples, we used the Flory-Rehner theory [27] in its modified form by Peppas and Merrill [28]. The measured masses of the hydrogels right after fabrication $\left(\mathrm{m}_{\mathrm{c}}\right)$, in equilibrium swelling $\left(\mathrm{m}_{\mathrm{s}}\right)$, and in their dry state $\left(\mathrm{m}_{\mathrm{d}}\right)$ were used to calculate the polymer volume fraction of the hydrogels in their swollen $\left(v_{2, s}\right)$ and relaxed $\left(v_{2, r}\right)$ states as well as their estimated mesh sizes $\xi$. Details of the calculation can be found in the Supplementary Information. Since this model is not well suited to determine quantitatively the properties of hydrogel co-networks [29], we studied the influence of PEGDA within the GelMA networks by analysing the diffusion profiles of dextran fluorescent molecules of different molecular weights as they passed through the hydrogels. GelMA and GelMA-PEGDA hydrogel discs (6.5 $\mathrm{mm}$ in diameter and $1 \mathrm{~mm}$ in height) were fabricated on top of porous PET membranes ( $5 \mu \mathrm{m}$ pore size) using a UV dose of $1.88 \mathrm{~J} \cdot \mathrm{cm}^{-2}$. Hydrogels were mounted on 24 -well polycarbonate Transwell ${ }^{\circledR}$ filter inserts (Corning Costar) using double-sided pressure-sensitive adhesive rings (Adhesives Research) as described elsewhere [26]. Then, $200 \mu \mathrm{L}$ of solutions containing dextrans at $0.25 \mathrm{mg} \cdot \mathrm{mL}^{-1}$ in PBS were loaded in the apical chambers while adding $600 \mu \mathrm{L}$ of PBS to the basolateral chambers. Dextran molecules of $4 \mathrm{kDa}$ (FITCDextran), $70 \mathrm{kDa}$ (Rhodamine-Dextran), and $500 \mathrm{kDa}$ (FITCDextran) (all from Sigma-Aldrich) were used separately. The Transwell ${ }^{\circledR}$ plates were incubated at $37^{\circ} \mathrm{C}$. At regular intervals (from 0 to $240 \mathrm{~min}$ ), $50 \mu \mathrm{L}$ were sampled from the basolateral compartments and replaced with $50 \mu \mathrm{L}$ of PBS. Collected samples were transferred to 96 black well plates and FITC or Rhodamine fluorescence was measured at excitation/emission wavelengths of $490 / 525 \mathrm{~nm}$ and $540 / 625 \mathrm{~nm}$, respectively, using an Infinite M200 PRO Multimode microplate reader (Tecan). The changing concentration of dextrans over time was determined using standard calibration curves. Apparent diffusion coefficients $\left(\mathrm{D}_{\mathrm{app}}\right)$ for each dextran were calculated as

$$
D_{a p p}=\frac{P_{a p p} \cdot h}{K}
$$

where $\mathrm{h}$ is the height of the hydrogels, $\mathrm{K}$ the hydrogel/water partition coefficient (assumed to be 1), and $\mathrm{P}_{\text {app }}$ the apparent permeability. $\mathrm{P}_{\text {app }}$ was obtained from the change in cumulative diffusion of the dextran over time (Figure S3) [30]. The effects of PEGDA on the network structure's mesh size were evaluated by comparing the apparent diffusion coefficients of 
the GelMA-PEGDA hydrogel co-networks to their GelMA counterparts (with equivalent macromer compositions).

\subsection{Mechanical properties analysis}

The mechanical properties of hydrogels are important physical cues that direct cell behaviour. For their measurement, we fabricated discs on top of coverslips covered with a porous PET membrane. Pools, $10 \mathrm{~mm}$ in diameter and about $3 \mathrm{~mm}$ in height, were photopolymerized with an energy dose of 3.76 $\mathrm{J} \cdot \mathrm{cm}^{-2}$ (double the standard dose to ensure photopolymerization across the entire thickness). After swelling in PBS, hydrogel diameters were no longer uniform and had to be re-punched to obtain consistent $10 \mathrm{~mm}$ diameter samples. Sample heights were accurately determined using a high precision Mitutoyo calliper (Mitutoyo Corporation). A Zwick-Roell Z0.5TN testing machine (Zwick Roell Group) was used to obtain stress-strain curves from compression assays at room temperature. Stress-strain curves were recorded limiting the strain rate to $5 \% \mathrm{~min}^{-1}$ and the maximum strain to $50 \%$. An initial load of $5 \mathrm{mN}$ was applied to facilitate proper contact between the hydrogels and the compression plates. The apparent elastic modulus of the samples was determined using TestXpert II, v.3.41 analysis software from the slope of the linear region of the stress-strain curves, corresponding to a strain of $10-20 \%$.

\subsection{Degradation analysis}

To investigate the degradation properties of GelMA and GelMA-PEGDA co-networks, we fabricated disc-shaped hydrogels (10 $\mathrm{mm}$ in diameter and $1 \mathrm{~mm}$ in thickness) on top of untreated cover glasses by applying a UV exposure dose of $3.00 \mathrm{~J} \cdot \mathrm{cm}^{-2}$. After fabrication, hydrogels were detached from the cover glasses and left to swell in an Eppendorf tube with 1 $\mathrm{mL}$ of PBS. After swelling, PBS was replaced by $1 \mathrm{~mL}$ of collagenase type II $2.5 \mathrm{U} / \mathrm{mL}$ solution (Sigma-Aldrich) and incubated at $37^{\circ} \mathrm{C}$ for 96 hours while regularly removing collagenase solution by centrifuging the samples for $5 \mathrm{~min}$ at $8000 \mathrm{rpm}$. The undigested hydrogels were washed twice by adding Milli-Q water and centrifuged before decanting the supernatant. Then, the hydrogels were freeze-dried (Freeze Dryer Alpha 1-4 LD Christ) and weighed. The mass remaining percentage was determined by comparing the mass before $(\mathrm{t}=0)$ and after $(\mathrm{t})$ enzyme incubation:

$$
\text { Mass remaning }(\%)=\frac{M_{(t)}}{M_{(t=0)}} \cdot 100
$$

\subsection{Fabrication and characterization of cell-laden GeIMA-PEGDA hydrogels}

NIH-3T3 fibroblasts (ATCC ${ }^{\circledR}$ CRL-1658 ${ }^{\mathrm{TM}}$ ) were used as cell model to mimic the stromal compartment of the intestinal tissue. Fibroblasts were expanded and maintained in $75 \mathrm{~cm}^{2}$ flasks in high glucose DMEM (Gibco, Thermo Fisher Scientific), supplemented with $10 \% \mathrm{v} / \mathrm{v}$ fetal bovine serum (FBS) (Gibco, Thermo Fisher Scientific) and $1 \% \mathrm{v} / \mathrm{v}$ Penicillin/Streptomycin (Sigma-Aldrich). NIH-3T3 were grown in an incubator at $37^{\circ} \mathrm{C}$ and $5 \% \mathrm{CO}_{2}$ (exchanging the medium every other day) and passaged twice a week. To produce cell-laden hydrogels, NIH-3T3 $\left(5 \cdot 10^{6}\right.$ cells $\left.\cdot \mathrm{mL}^{-1}\right)$ were trypsinised and re-suspended in pre-polymer solutions containing the polymers and $0.5 \% \mathrm{w} / \mathrm{v}$ Irgacure 2959 photoinitiator in DMEM supplemented with $1 \% \mathrm{v} / \mathrm{v}$ Penicillin/Streptomycin. DMEM without Phenol red was employed to avoid potential effects during photopolymerization. Cell-containing polymer solutions were kept at $37^{\circ} \mathrm{C}$ before polymerization. Hydrogels $(6.5 \mathrm{~mm}$ in diameter and $0.5 \mathrm{~mm}$ high) were fabricated on silanized PET membranes ( $5 \mu \mathrm{m}$ pore size) (Figure 2(a)), by exposing them to a UV dose of $1.88 \mathrm{~J} \cdot \mathrm{cm}^{-2}$. Unreacted polymer and photoinitiator were washed out with warm cell culture medium supplemented with $10 \%$ of Penicillin/Streptomycin and $1 / 250$ of normocin (Invitrogen). Cell-laden hydrogels were then attached to Transwell ${ }^{\circledR}$ inserts using PSA rings and cultured for 7 days in an incubator at $37^{\circ} \mathrm{C}$ and $5 \% \mathrm{CO}_{2}$, exchanging the medium every other day. L-Ascorbic acid at $50 \mu \mathrm{g} \mathrm{mL}^{-1}$ (Sigma Aldrich) was added every other day to the cell culture medium to stimulate the production of extracellular matrix [31].

Cell viability on cell-laden hydrogels was investigated using a calcein-AM/ethidium homodimer Live/Dead kit (Invitrogen) 1 and 7 days after cell encapsulation and monitored via confocal laser scanning microscope (LSM 800, Zeiss). Image processing and cell viability quantification was performed manually using ImageJ v.1.49b software (http://rsb.info.nih.gov/ij, NIH) to determine the percentage of viable cells at each time point.

\subsection{Fabrication and functional characterization of $a 3 D$ model of the intestinal mucosa}

2.7.1 Fabrication of the 3D model. Fibroblast-laden GelMA-PEGDA hydrogels were produced as described above to generate a model of the intestinal stromal compartment. Immediately after fabrication, Caco-2 cells $\left(\mathrm{ATCC}^{\circledR}\right.$ HTB$37^{\mathrm{TM}}$ ) were seeded on top of the hydrogels to represent the epithelial component of the tissue. Caco-2 cells were maintained in $75 \mathrm{~cm}^{2}$ flasks in high glucose DMEM (Gibco, Thermo Fisher Scientific), supplemented with $10 \% \mathrm{v} / \mathrm{v}$ fetal bovine serum (FBS) (Life Technologies), 1\% v/v Penicillin/Streptomycin (Sigma Aldrich), and 1\% v/v of nonessential amino acids (Gibco, Thermo Fisher Scientific). Cells were kept in an incubator at $37^{\circ} \mathrm{C}$ and $5 \% \mathrm{CO}_{2}$ (exchanging 
the medium every other day) and passaged weekly. Then, Caco-2 cells were seeded at a density of $7.5 \cdot 10^{5}$ cells $\cdot \mathrm{cm}^{-2}$ on the fibroblast-laden $7.5 \% \mathrm{w} / \mathrm{v}$ GelMA-5\% w/v PEGDA hydrogel discs $(6.5 \mathrm{~mm}$ in diameter, $0.5 \mathrm{~mm}$ high) fabricated onto PET porous membranes and mounted on Transwell ${ }^{\mathbb{B}}$ inserts. Control experiments were performed by seeding Caco-2 cells on fibroblast-free hydrogels with the same composition. Additionally, Caco-2 cells were seeded on standard 24-well polycarbonate Transwell ${ }^{\circledR}$ filter inserts $(0.4$ $\mu \mathrm{m}$ pore size) at a density of $7.5 \cdot 10^{5}$ cells $\cdot \mathrm{cm}^{-2}$. Cells were cultured for 21 days, exchanging the medium every other day. The medium composition used for the co-culture was identical to the one used for the Caco-2 cells with the addition of Lascorbic acid every other day.

\subsubsection{Immunofluorescence and histological analysis.}

The characteristic morphology and polarization of Caco-2 cells forming an epithelial barrier were studied by immunostaining after culturing for 21 days. Samples were fixed with $10 \%$ neutral buffered formalin solution (SigmaAldrich) at $4 \mathrm{C}$ for 1 hour, permeabilized with $0.5 \%$ Triton-X (Sigma-Aldrich) and blocked with $1 \%$ bovine serum albumin (Sigma-Aldrich), 3\% donkey serum (Millipore), and 0.3\% Triton-X. Primary antibodies against ZO-1 (Abcam) (2 $\left.\mu \mathrm{g} \cdot \mathrm{mL}^{-1}\right)$ and $\beta$-catenin (Abcam) $\left(1 \mu \mathrm{g} \cdot \mathrm{mL}^{-1}\right)$ were incubated overnight at $4^{\circ} \mathrm{C}$ under shacking conditions. Samples were then incubated with secondary antibodies and/or RhodaminePhalloidin (Cytoskeleton) $(0.07 \mu \mathrm{M})$ for $2 \mathrm{~h}$ at $4^{\circ} \mathrm{C}$ under shacking conditions. Anti-goat Alexa 488 (Invitrogen, Thermo Fisher Scientific) and anti-rabbit Alexa 647 (Invitrogen, Thermo Fisher Scientific) $\left(4 \mu \mathrm{g} \cdot \mathrm{mL}^{-1}\right)$ were used as secondary antibodies. Finally, samples were incubated with DAPI (Thermo Fisher Scientific) $\left(5 \mu \mathrm{g} \cdot \mathrm{mL}^{-1}\right)$ for $30 \mathrm{~min}$.

For the hydrogel-embedded fibroblasts, cell morphology and extracellular matrix proteins production were analysed by immunofluorescence, following the same protocol described above. After fixation, permeabilization, and blocking steps, primary antibodies against Collagen IV (Biorad) $\left(1.6 \mu \mathrm{g} \cdot \mathrm{mL}^{-}\right.$ $\left.{ }^{1}\right)$ were incubated overnight at $4^{\circ} \mathrm{C}$ under shacking conditions. Then, secondary antibodies anti-goat Alexa 488 (Invitrogen, Thermo Fisher Scientific) $\left(4 \mu \mathrm{g} \cdot \mathrm{mL}^{-1}\right)$ and/or Rhodamine Phalloidin (Cytoskeleton) $(0.07 \mu \mathrm{M})$ were incubated for $2 \mathrm{~h}$ at $4^{\circ} \mathrm{C}$ under shacking conditions. Finally, samples were incubated with DAPI $\left(5 / \mu \mathrm{g} \cdot \mathrm{mL}^{-1}\right)$ for $30 \mathrm{~min}$. After immunostaining, hydrogels were mounted facing downward onto glass coverslips and imaged using a confocal laserscanning microscope (LSM 800, Zeiss). $500 \mu \mathrm{m}$ PDMS spacers were employed to prevent sample damage. A drop of Fluoromount G (Southern Biotech) was added to preserve the dyes and prevent the sample from drying. Acquired Z-stacks were processed using Image $\mathrm{J}$ software. For a better visualization of the embedded cells across the entire height of the hydrogel, stained hydrogels were embedded and cross- sectioned using the optimal cutting temperature (OCT) technique. First, samples were incubated overnight with $30 \%$ sucrose (Sigma-Aldrich) at $4^{\circ} \mathrm{C}$, then embedded in OCT (Tissue-Tek $^{\circledR}$ O.C.T. Compound, Sakura ${ }^{\circledR}$ Finetek). OCT tissue sections ( $\sim 7 \mu \mathrm{m}$ in thickness) were cut, air dried, and stored at $-80^{\circ} \mathrm{C}$ for further analysis. Alternatively, some samples were embedded in paraffin for later hematoxylineosin staining. These samples were embedded in an automatic tissue processor machine (Tissue Tek VIP, Sakura) following routine procedures and resulting in paraffin-embedded sections of about $3 \mu \mathrm{m}$ thick. After being cut and air dried, these samples were further dried over-night at $60^{\circ} \mathrm{C}$ and then stored at room temperature before hematoxylin and eosin staining. For later imaging, both types of hydrogel slices were mounted on a glass coverslip using Fluoromount $\mathrm{G}$ (Southern Biotech) and visualized using a confocal laser-scanning microscope (LSM 800, Zeiss).

\subsubsection{Transepithelial electrical resistance and} permeability studies. The integrity of the barrier developed by the epithelial cells on the fibroblast-laden hydrogels and control samples was monitored every two days throughout the culture period (21 days). For this purpose, the transepithelial resistance (TEER) between the two compartments of the Transwell ${ }^{\circledR}$ chambers was measured using an EVOM2 Epithelial voltohmmeter with an STX3 electrode (World precision Instruments). Measured resistance values were corrected by subtracting the resistances of the porous PET membranes and the hydrogels. TEER values were normalized to the total surface area of the epithelial monolayers. After 21 days, permeability was measured using fluorescent-labelled dextrans (4 kDa FITC dextran,FD4, and $70 \mathrm{kDa}$ rhodamine dextran, FD70) as model compounds, and insulin as biological model drug. In brief, cells were washed with DMEM without Phenol red (Gibco, Life Technologies) and $200 \mu \mathrm{L}$ of the test solutions containing $0.5 \mathrm{mg} \cdot \mathrm{mL}^{-1}$ dextrans or $0.25 \mathrm{mg} \cdot \mathrm{mL}^{-1}$ insulin were added to the apical side of the Transwell ${ }^{\mathbb{B}}$ inserts. Then, $600 \mu \mathrm{L}$ of DMEM without Phenol red were added to the basolateral compartments. Samples were collected from the basolateral compartments at given time points followed by buffer replacement. During the experiment, cells were incubated at $37^{\circ} \mathrm{C}$ on a horizontal shaker at $50 \mathrm{rpm}$. Samples were then analysed by measuring the fluorescence using an Infinite M200 PRO Multimode microplate reader (Tecan) or by an insulin ELISA kit (Mercodia). The apparent permeability $\left(\mathrm{P}_{\text {app }}\right)$ was determined from the change in cumulative diffusion of the dextran over time [30].

2.7.8 Epithelial barrier disruption. The impact of the stromal cells on barrier recovery after tight-junction disruption was studied after 21 days of culturing by disrupting the barrier with ethylenediaminetetraacetic acid (EDTA) (Sigma- 
Aldrich). $5 \mathrm{mM}$ solution of EDTA in high glucose DMEM supplemented with $10 \% \mathrm{v} / \mathrm{v}$ fetal bovine serum (FBS), $1 \% \mathrm{v} / \mathrm{v}$ Penicillin/Streptomycin and $1 \% \mathrm{v} / \mathrm{v}$ of non-essential amino acids, were added to the apical $(200 \mu \mathrm{L})$ and basolateral $(600$ $\mu \mathrm{L}$ ) compartments. After 5 min of incubation, samples were washed with PBS and stored at $37^{\circ} \mathrm{C}$ in DMEM, measuring TEER values at given time points.

\subsection{Data analysis and statics}

The data are presented as the mean \pm standard deviation (SD). The graphs were plotted using OriginPro 8.5 software (OriginLab). In the case of normal distributions, differences between groups were compared through a one-way analysis of variance (ANOVA). Turkey's test was performed as indicated in the figure legends. Differences were considered as statistically significant if $\mathrm{p}<0.05$.

\section{Results}

\subsection{Photopolymerized GelMA and GeIMA-PEGDA co- networks provide hydrogels with tuneable network properties}

Nuclear magnetic resonance (NMR) spectroscopy of the methacrylation of the synthesized GelMA (Figure S1) yield bands at $5.3 \mathrm{ppm}$ and the $5.6 \mathrm{ppm}$ in the spectra of the GelMA samples, characteristic of the double bonds of the methacrylate groups. Additionally, the decrease of a band at $3.0 \mathrm{ppm}$ reveals the reaction of the methacrylic anhydride (MA) with the $\varepsilon$-amino groups from the Lysines in the gelatine [24]. These results confirm the success of the gelatine methacrylation process. By using the Habeeb Method [25], we found that adding $5 \% \mathrm{v} / \mathrm{v}$ of MA to the gelatine resulted in a polymer with a high degree of methacrylation $(76.7 \pm 2.1 \%)$. This parameter represents the percentage of $\varepsilon$-amino groups that are modified by the reaction, which determine the relative influence of the physical and chemical crosslinking mechanisms and, in turn, the mechanical properties of the final hydrogel [32]. As mechanical properties have been identified as critical cues affecting cellular behaviour [33], polymers with high degrees of methacrylation promoting chemical crosslinking are claimed to provide a more mechanically stable and cell-friendly environment [18].

GelMA, PEGDA and GelMA-PEGDA polymeric solutions were mixed with a photoinitiator (Irgacure 2959) placed in circular PDMS pools and crosslinked by $U \mathrm{~V}$, using exposure doses from 1 to $3.76 \mathrm{~J} \cdot \mathrm{cm}^{-2}$ (Figure 1(a) and 1(b)) to form discshaped hydrogels. With this exposure conditions, only PEGDA polymers exhibited a complete lack of cross-linking at the macromer concentrations used. For all the solutions tested, UV dose of less than $1 \mathrm{~J} \cdot \mathrm{cm}^{-2}$ lead to poorly crosslinked networks or no crosslinking at all.
GelMA has been shown to precipitate in GelMA-PEGDA co-networks when using high concentrations of PEG polymers of high molecular weight [34]. Through microscope imaging analysis of the distribution of fluorescence labelled GelMA chains we verified that GelMA precipitations did not occur in our composite hydrogel. We also did not observe any phasesegregation indicating the formation of homogeneous conetworks during the polymerization process (Figure S2).

The swelling properties of polymeric networks depend on both the pore size and the polymer-solvent interaction and are important as they can affect the diffusive and mechanical properties of the material $[20,26,35]$. In here, hydrogel swelling as a function of the total macromer concentration of the polymer $(7.5 \% \mathrm{w} / \mathrm{v}$ or $12.5 \% \mathrm{w} / \mathrm{v})$, showed that a higher macromer content led to significant decreases in swelling ratios for both the GelMA and GelMA-PEGDA samples (Figure 1(c)). This is a consequence of denser network structures that have higher crosslinking densities. In addition, the incorporation of PEGDA has a strong impact on the swelling properties of the co-network, increasing the hydrogels ability to attract and store water (Figure 1(c)). This effect, attributed to the higher hydrophilicity of the PEGDA polymeric chains, was more evident in samples with a lower macromer content (swelling ratio increased by $100 \%$ when adding PEGDA on $7.5 \% \mathrm{w} / \mathrm{v}$ samples and only by $54 \%$ when adding PEGDA on $12.5 \% \mathrm{w} / \mathrm{v}$ samples). This result is in agreement with previous findings [17].

Diffusivity analyses of dextran compounds of different molecular size were performed to gain insight on the porous nature of the hydrogels. Our results showed that the permeability of large to medium sized molecules such as FD500 and FD70 with molecular weights of 500 and $70 \mathrm{kDa}$ and hydrodynamic diameters of 32 and $11.6 \mathrm{~nm}$, respectively [36], were hindered by the hydrogels, while the smaller FD4 with $4 \mathrm{kDa}$ of molecular weight and $2.8 \mathrm{~nm}$ of hydrodynamic diameter [37] could diffuse more easily (Figure S3). This effect was consistent for all the different hydrogels compositions (Figure 1(d)). For FD4, the diffusion coefficient decreased with increasing macromolecular content. This behaviour agrees with experimental diffusivity coefficients measured for GelMA networks [38]. We computed the values for the mesh size of PBS-swollen GelMA networks following Peppas and Merrill modifications proposed for the FloryRehner theory [27] (see supplementary Material and Methods), and found that those decreased from about $21 \mathrm{~nm}$ to about $16 \mathrm{~nm}$ when the macromer content was increased from 7.5 to $12.5 \% \mathrm{w} / \mathrm{v}$. PEGDA-containing hydrogels did not show significant differences in diffusivity properties with respect to their GelMA counterparts (Figure 1(d)), which suggests that mesh size is unaffected by the addition of PEGDA. 
The mechanical properties of GelMA and GelMA-PEGDA hydrogels were evaluated in compression assays. The stressstrain curves recorded did not show any sample failure, even at the maximum strain level of $50 \%$. This demonstrates that all hydrogels have a linear behavior as elastomers within the load range and the conditions of the assay. The apparent elastic modulus significantly increased for higher macromer content, both in the GelMA and GelMA-PEGDA samples (Figure 1(e)), as would be expected for denser networks. For identical macromer contents, the addition of PEGDA led to a decrease in the apparent elastic modulus, particularly $(>90 \%$ decrease) for low $(7.5 \% \mathrm{w} / \mathrm{v})$ macromer content.
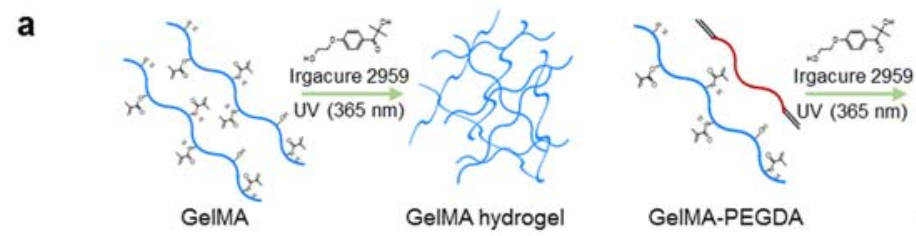

b
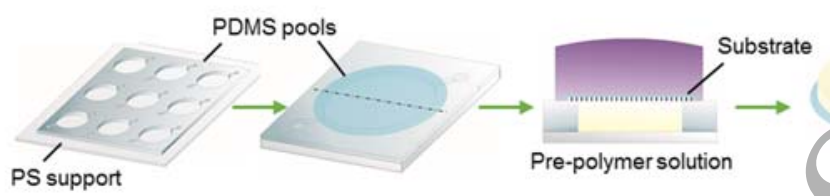

C

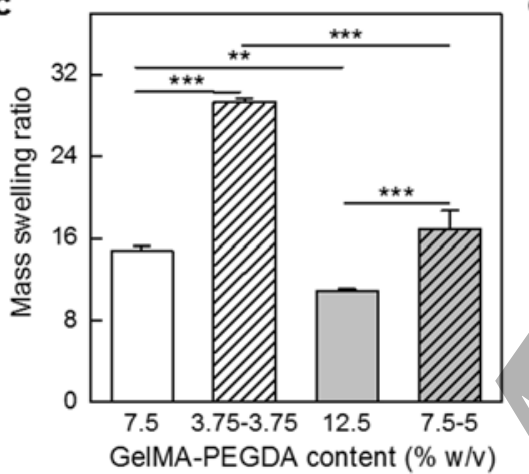

d

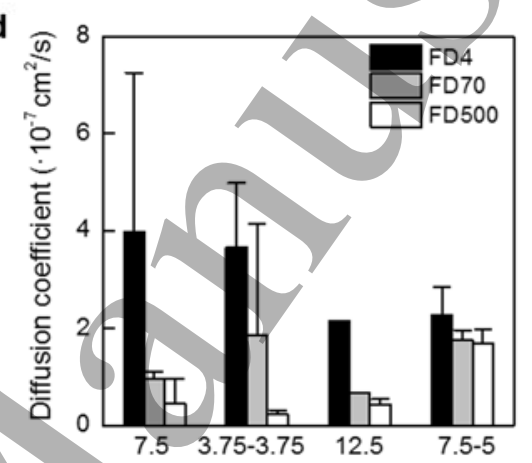

$$
\text { e }
$$
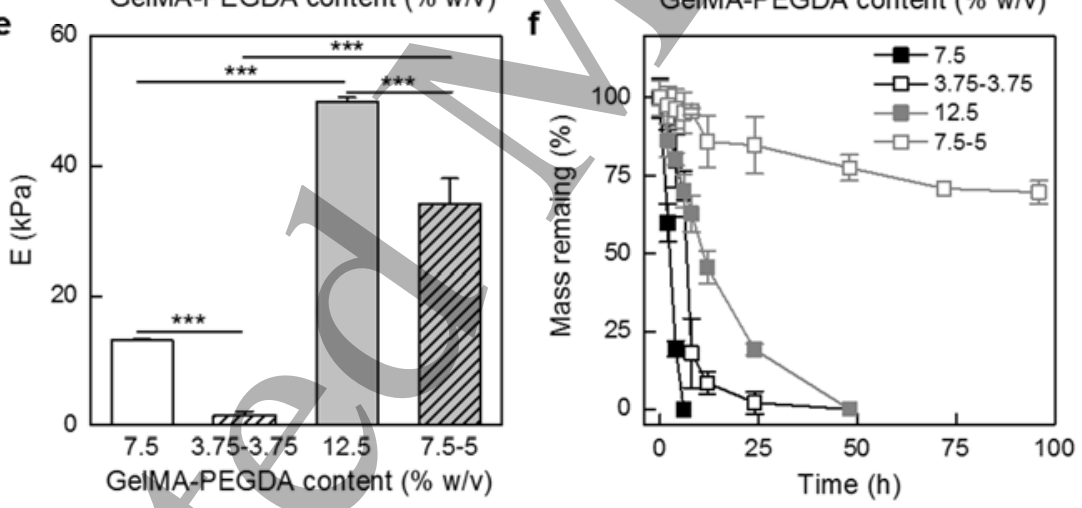

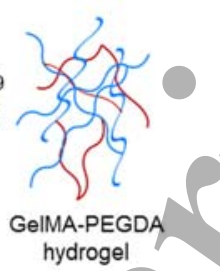

Figure 1. (a) Schematic of the GelMA and GelMA-PEGDA photo-polymerization reaction and co-networks formed. (b) Schematic of the single-step photo-crosslinking process employed. Pre-polymer solution was poured into a PDMS pool and covered with a cover glass or a porous membrane which both act as substrates. UV exposure produces either a single or an array of photo-crosslinked hydrogel networks. (c) Mass swelling ratio, (d) diffusion coefficient for different molecule sizes $(F D 500=500 \mathrm{kDa}$, with a hydrodynamic diameter of $32 \mathrm{~nm}$; FD70 $=70 \mathrm{kDa}$, hydrodynamic diameter of $11.6 \mathrm{~nm}$; FD4 $4 \mathrm{kDa}$, hydrodynamic diameter of $2.8 \mathrm{~nm}$ ), (e) apparent elastic modulus, E, and (f) degradation upon collagenase incubation (shown as mass remaining with time), for different GelMA networks and GelMA-PEGDA co-networks: 7.5 (7.5\% w/v GelMA), 3.753.75 (3.75\% w/v GelMA-3.75\% w/v PEGDA), 12.5 (12.5\% w/v GelMA), 7.5-5 (7.5\% w/v GelMA-5\% w/v PEGDA). Values are shown as mean $\pm \mathrm{SD}(\mathrm{n}=3) . * * \mathrm{p}<0.005$ and $* * * \mathrm{p}<0.001$.

The effects of adding PEGDA agree with those produced by a less dense network that allows higher water storage. Therefore, by co-polymerizing GelMA with PEGDA, the mechanical properties have become tuneable (here with apparent elastic moduli ranging from 2 to $50 \mathrm{kPa}$ ) and comparable to those reported for soft tissues in vivo (1-100 
$\mathrm{kPa}$ ) [39]. We next investigated the effect of having GelMAPEGDA co-networks on hydrogel degradation by incubating the different samples with collagenase. Our results showed that GelMA hydrogels containing $7.5 \%$ and $12.5 \% \mathrm{w} / \mathrm{v}$ of macromer were completely degraded after 4 and 24 hours, respectively (Figure 1(f)). We also found that for a given macromer content, the addition of PEGDA to form GelMAPEGDA hydrogel co-networks retarded the degradation.

Samples containing $7.5 \% \mathrm{w} / \mathrm{v}$ of GelMA and $5 \% \mathrm{w} / \mathrm{v}$ of PEGDA were only $30 \%$ degraded after $96 \mathrm{~h}$ of collagenase

a
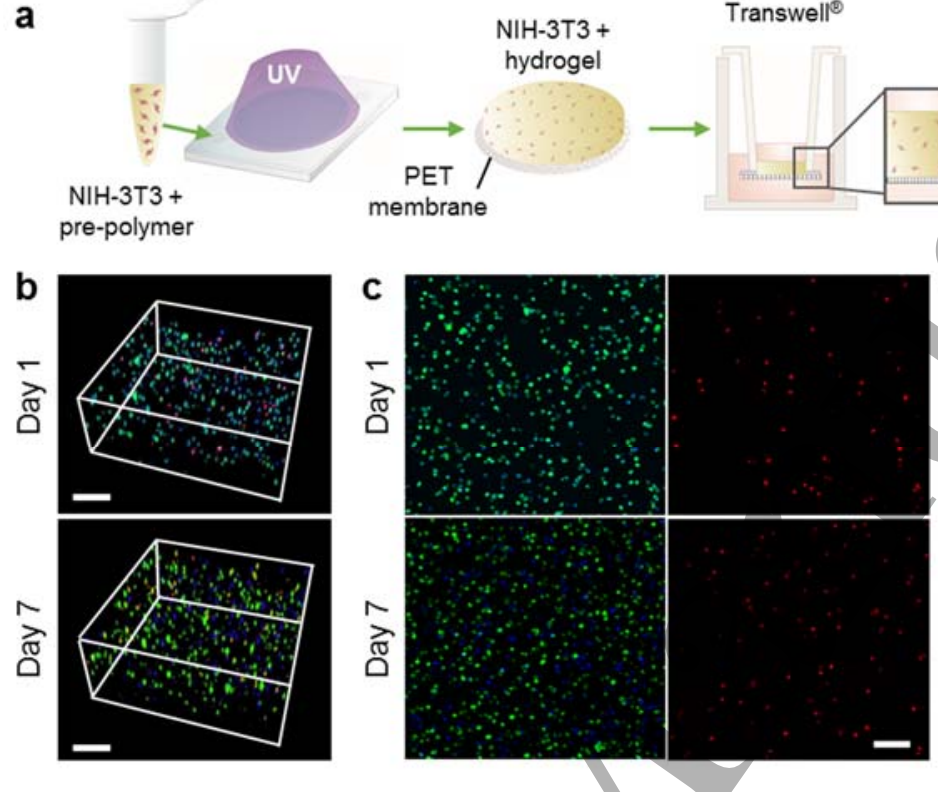

e
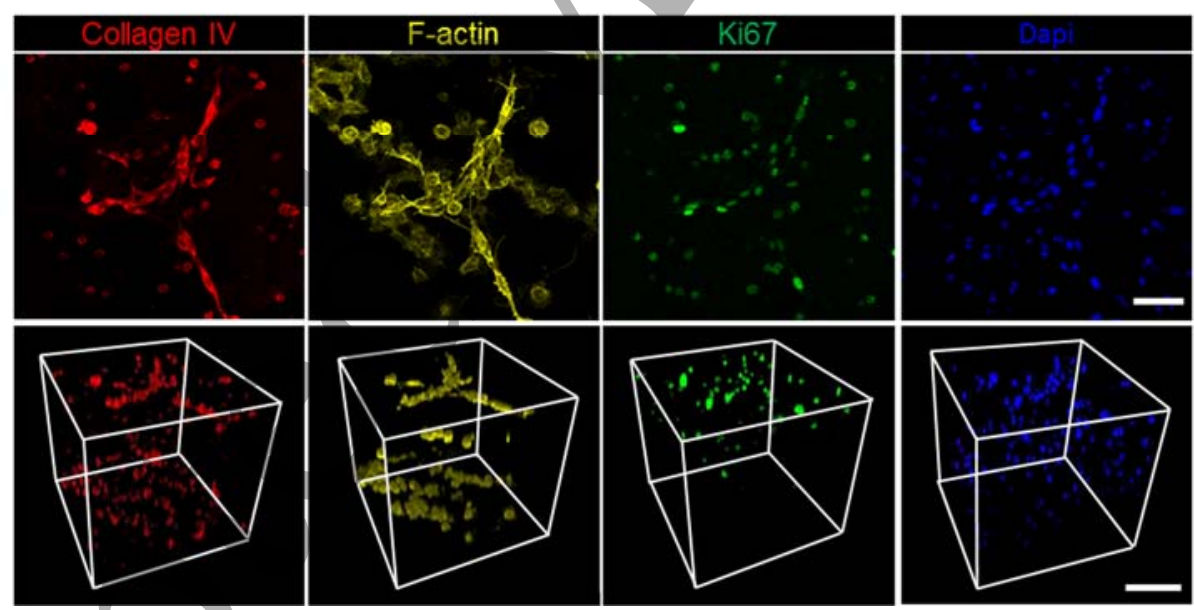

Figure 2. (a) Schematic to illustrate the NIH-3T3 fibroblast encapsulation process in the hydrogel co-network. (b) Confocal 3D reconstruction of the hydrogel co-network showing the spatial distribution of the NIH-3T3 embedded cells on days 1 and 7 after live/dead assays (live cells stain in green, dead cells in red). Dapi was used to stain the nuclei. Scale bars: $200 \mu \mathrm{m}$. (c) Maximum intensity projections of samples shown in panel (b). Scale bar: $100 \mu \mathrm{m}$. (d) Quantification of cell viability at days 1 and 7 after encapsulation based on live/dead staining. Values are the mean percentage of cell viability $\pm \operatorname{SD}(n=3), * * * p<0.001$. (e) Immunostaining for Collagen IV, F-actin, and Ki67 of hydrogel co-networks with embedded NIH-3T3 fibroblasts after 7 days in culture shown as maximum intensity projections (top panels) and confocal 3D reconstruction (bottom panels). Scale bars: $50 \mu \mathrm{m}$ (top) and $100 \mu \mathrm{m}$ (bottom panels). 
Overall, our GelMA-PEGDA co-networks are thus highly tuneable matrices in which water content, pore size, mechanical properties, and degradability can be tailored to suit the requirements of tissue-specific microenvironments

\subsection{GelMA-PEGDA hydrogel co-networks support fibroblast $3 D$ cultures}

Based on the degradation results for the GelMA and GelMAPEGDA samples and the long culture times required for the proper formation of intestinal epithelial cells (usually 3 weeks) [40], we selected the co-networks formed by $7.5 \%$ GelMA $5 \%$ PEGDA for the cellular experiments. NIH-3T3 fibroblasts were mixed with the GelMA-PEGDA polymer solution and the photoinitiator before encapsulating them through exposure to a UV dose of $1.88 \mathrm{~J} \cdot \mathrm{cm}^{-2}$ (Figure 2(a)). Disc-shaped hydrogels $(6.5 \mathrm{~mm}$ in diameter and $0.5 \mathrm{~mm}$ thick) were initially loaded with $5 \cdot 10^{6}$ cells $\cdot \mathrm{mL}^{-1}$ cells. The hydrogel thickness was selected by considering that cell viability is limited by oxygen diffusion and computing the maximum hydrogel thickness for cell survival for NIH-3T3 cells ( $3 \mathrm{~mm}$ considering $2 \cdot 10^{7}$ cells $\left.\cdot \mathrm{mL}^{-1}\right)$ [5]. The samples were fabricated on porous membranes and mounted on Transwell ${ }^{\mathbb{P}}$ inserts (Figure 2(a)).

Cell viability of the encapsulated cells was evaluated through live/dead assays and confocal microscopy. One day after encapsulation, cells were evenly distributed throughout the hydrogel and, although there were some non-viable cells (stained in red), the majority were alive (stained in green) (Figure 2(b, c)). Overall, cell viability, computed as the ratio between alive and dead cells, was greater than $85 \%$ right after encapsulation (Figure 2(d)). It has been previously shown that short-term cell viability after cell encapsulation decreases over time due to the stress induced by the photo-crosslinking process (UV irradiation, presence of radical species), and to the swelling caused by the incubation with cell culture medium.

To evaluate these effects on our hydrogels, we analysed cell viability of the encapsulated cells at later culture time points. We found that at 7 days of culture, the cells were still evenly distributed along the thickness of the hydrogels (Figure 2(b), lower panel) and only a not statistically significant decrease of less than $10 \%$ in cell viability was observed (Figure 2(c), lower panel and Figure 2(d)). After 14 and 21 days of culture, cell viability had decreased considerably with most viable cells being located at or near the surface of the hydrogels (Figure S4). These results agree with previous findings where 3D environments have been reported to decrease cell metabolic activity and induce growth arrest, compared to 2D equivalents, leading to a metabolic steady state when cultured for long periods [16,41]. We next tested whether fibroblasts embedded in GelMA-PEGDA hydrogels were functional secreting ECM proteins. Specifically, we analysed the expression of collagen IV as relevant ECM protein of the stromal compartment and epithelial basement membrane. Immunostaining showed that embedded fibroblasts cultured for 7 days had spread thorough the hydrogel and were actively producing collagen IV (Figure 2(e)). In addition, embedded fibroblasts tested positive for the Ki67 proliferation marker after 7 days (Figure 2(e)). Fibroblasts that were growing closer to the surface of the hydrogel proliferated more and exhibit a broader spread morphology compared to cells located in the interior of the hydrogel (Figure 2(e), bottom panels). We hypothesize that these characteristics could be associated to hypoxic conditions and mass-transport issues inherent to the 3D cell culture microenvironment [39]. To test whether this limitation is particular to our GelMA-PEGDA hydrogel conetwork formulation or whether it might be overcome in future experiments, we conducted a pilot experiment with cellladen hydrogels cultured in custom-made bioreactors, previously developed in our laboratory, with medium perfusion for 21 days [42]. We observed that the viable fibroblasts were homogenously distributed throughout the entire hydrogel when cultured in this bioreactor (Figure S4). However, for practical reasons, we chose to continue with the static Transwell ${ }^{\mathbb{B}}$ set-up in the work reported here.

Overall, our results show that GelMA-PEGDA co-networks guarantee a high level of viability and secretory functions of the encapsulated fibroblasts for at least 7 days in culture, making them convenient candidates for developing the stromal component of 3D in vitro models of intestinal mucosa.

\subsection{GeIMA-PEGDA hydrogel co-networks mimic the epithelial and stromal components of the intestinal mucosa}

We next tested the capability of an epithelial intestinal cell line, Caco-2, to adhere and grow onto $7.5 \%$ GelMA - 5\% PEGDA hydrogel co-networks. For this purpose, disc-shaped hydrogels $6.5 \mathrm{~mm}$ in diameter and $0.5 \mathrm{~mm}$ in height were photo-crosslinked by applying a UV exposure dose of 1.88 $\mathrm{J} \cdot \mathrm{cm}^{-2}$. The samples were fabricated onto porous polymeric membranes and mounted in Transwell ${ }^{\circledR}$ inserts. We next seeded $7.5 \cdot 10^{5}$ Caco-2 cells $\cdot \mathrm{cm}^{-2}$ onto the hydrogels and followed their behaviour throughout the culture. Our results show that Caco-2 cells were able to adhere, spread, and proliferate on the surface of our hydrogels. After 21 days of culture, confocal microscopy images revealed the formation of a densely packed monolayer of Caco-2 cells covering the entire surface of the hydrogel (Figure S5). Visualising $\beta$ catenin and ZO-1 markers via immunofluorescence reveal the typical cobblestone-like shape of epithelial layers (Figure 3(b), upper panels), while the cross-sections (Figure 3(b), 
lower panels) show columnar, highly polarized cells exhibiting apical F-actin and ZO- 1 expression, with $\beta$-catenin expression confined to the basolateral side of the monolayers.
These results suggest that Caco-2 cells can form an epithelial monolayer on the GelMA-PEGDA hydrogel co-networks.

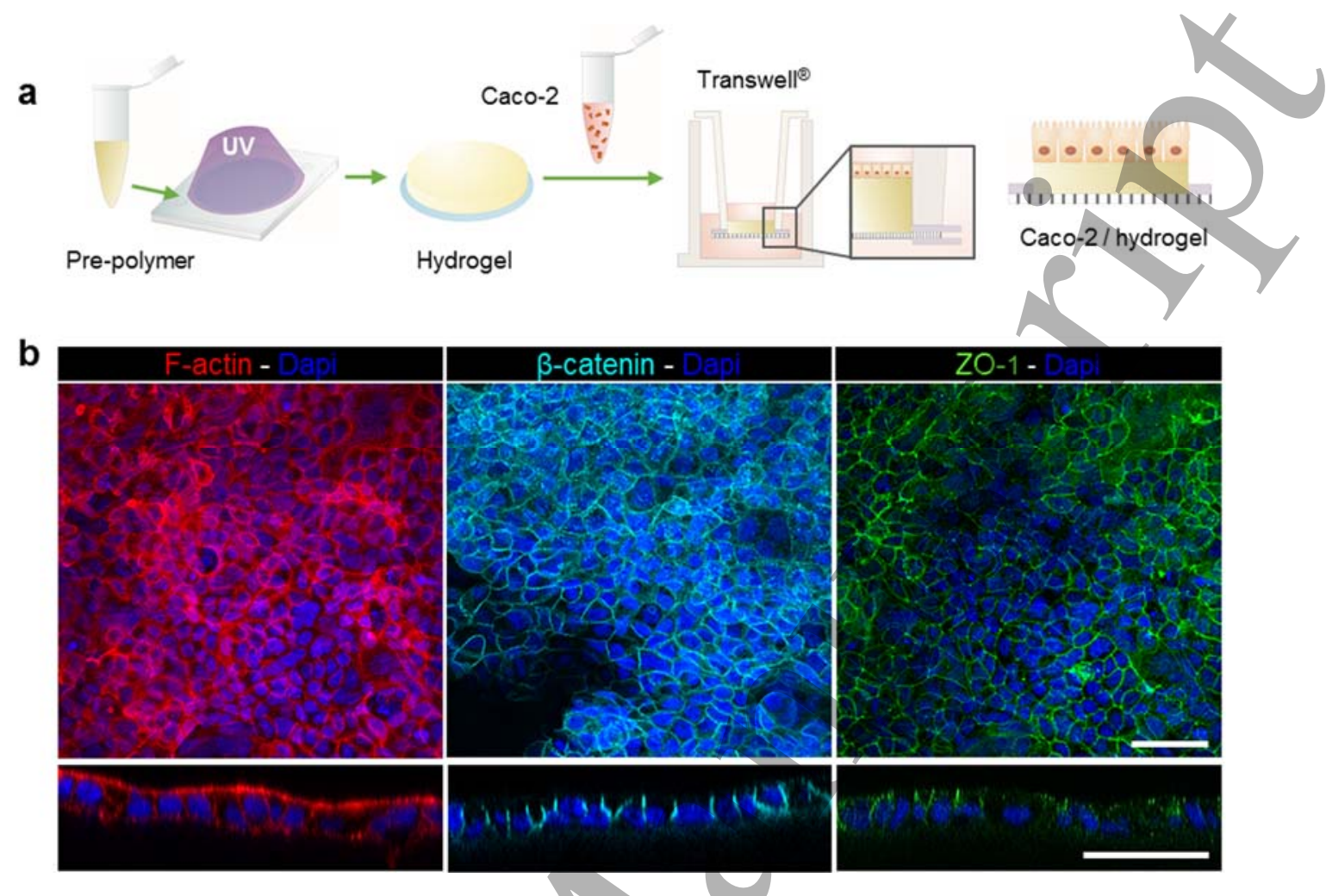

Figure 3. (a) Schematic to illustrate the Caco2 epithelial cells seeding method on the hydrogel co-network. (b) Maximum intensity projections of immunostainings for F-actin, $\beta$-catenin, and ZO-1 on the epithelial cell monolayer formed on top of the hydrogel co-networks (upper panels) and orthogonal sections (bottom panels). Dapi was used to stain the nuclei. Scale bars: 50 $\mu \mathrm{m}$.

By combining the fibroblast-laden GelMA-PEGDA hydrogels with the culture of epithelial monolayers sitting on top of them we then obtained an in vitro model of the intestinal mucosa. NIH-3T3 cells were encapsulated at a density of $5 \cdot 10^{6}$ cells $\cdot \mathrm{mL}^{-1}$ following the method described in the previous section to generate fibroblasts-laden hydrogels that were mounted in Transwell ${ }^{\circledR}$ inserts and seeded with Caco-2 cells as described before (Figure 4(a)). After 21 days of culture, the hydrogels showed good mechanical integrity and could be processed to perform histological studies. Hematoxylin-eosin staining of the construct's cross-sections about $3 \mu \mathrm{m}$ thick showed the formation of a continuous epithelial cell monolayer on top of the constructs and even distribution of fibroblasts throughout the hydrogel co-network (Figure 4(b)). Immunostaining showing apical F-actin and basolateral $\beta$-catenin confirmed the correct polarization of the epithelial monolayer formed on top of the mucosa-like hydrogel constructs. At this point in time, fibroblasts were mostly of a round shape while expressing collagen IV, which appears to have accumulated at the epithelial basement membrane (Figure 4(c)), suggesting their capacity to remodel the surrounding matrix. Based on these results, our GelMAPEGDA hydrogel co-networks appear as excellent candidates to produce long-lasting in vitro models of the intestinal mucosa that would allow to mimic in vivo interactions between stromal and epithelial cells under physiological and pathological conditions.

\subsection{Evaluation of the stromal component effect on the barrier properties of the intestinal mucosa model}

As a proof of concept, we employed our 3D model of intestinal mucosa to assess in vitro the effect of fibroblasts on the intestinal epithelial monolayer growth and barrier function. As functional hallmarks for epithelial barrier integrity and permeability, we measured the transepithelial electrical resistance (TEER) and the apparent permeability $\left(\mathrm{P}_{\mathrm{app}}\right)$ to paracellular model compounds of the epithelial monolayers grown under different experimental conditions. Hydrogel discs laden with fibroblasts were fabricated onto 
porous membranes, mounted on Transwell ${ }^{\circledR}$ inserts and seeded with Caco-2 cells following the methods earlier described. In addition, we used hydrogels discs without fibroblasts and seeded with Caco-2 cells, and Caco-2 cell monolayers grown directly on porous membranes, which is the current gold-standard culture format for these assays [43] (Figure 5(a)). Immunofluorescence images of the samples after 8 days of culture show that epithelial cells grown on fibroblast-laden hydrogels formed a continuous flat epithelial monolayer that fully covered the hydrogel surface. In contrast, on fibroblasts-free hydrogel samples the epithelial cells formed a discontinuous layer with dome-shaped structures (Figure 5(b)). TEER, which is directly related to the tightness of the epithelial barrier, increased in all samples including epithelial cells with increasing culture time (Figure 5(c))), indicating the formation of an epithelial monolayer with effective barrier.

a
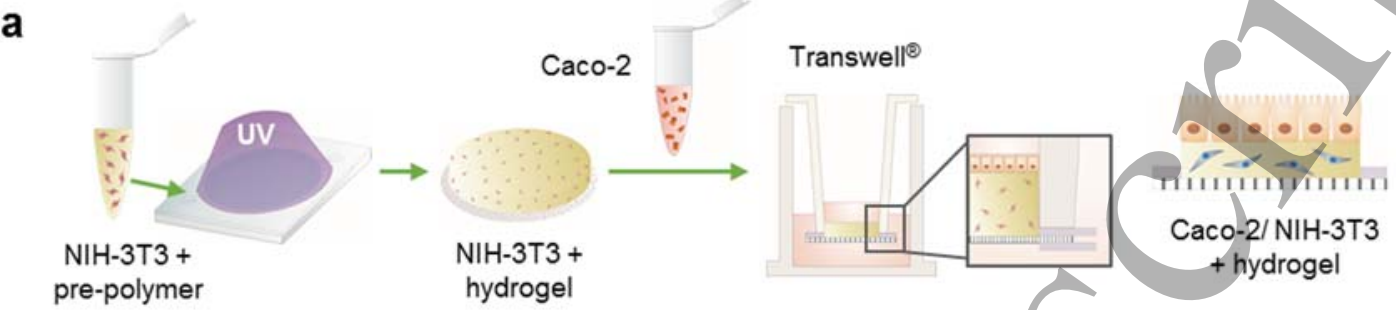

b
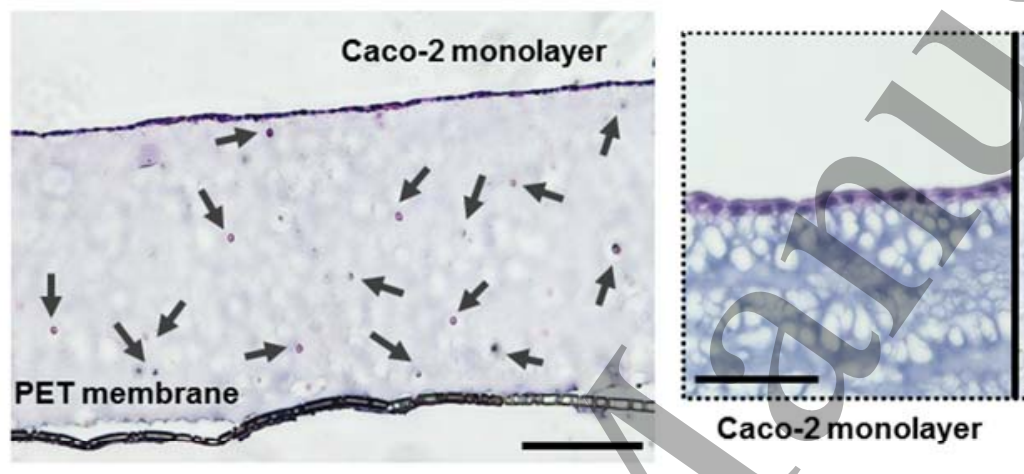

Caco-2monolayer

C
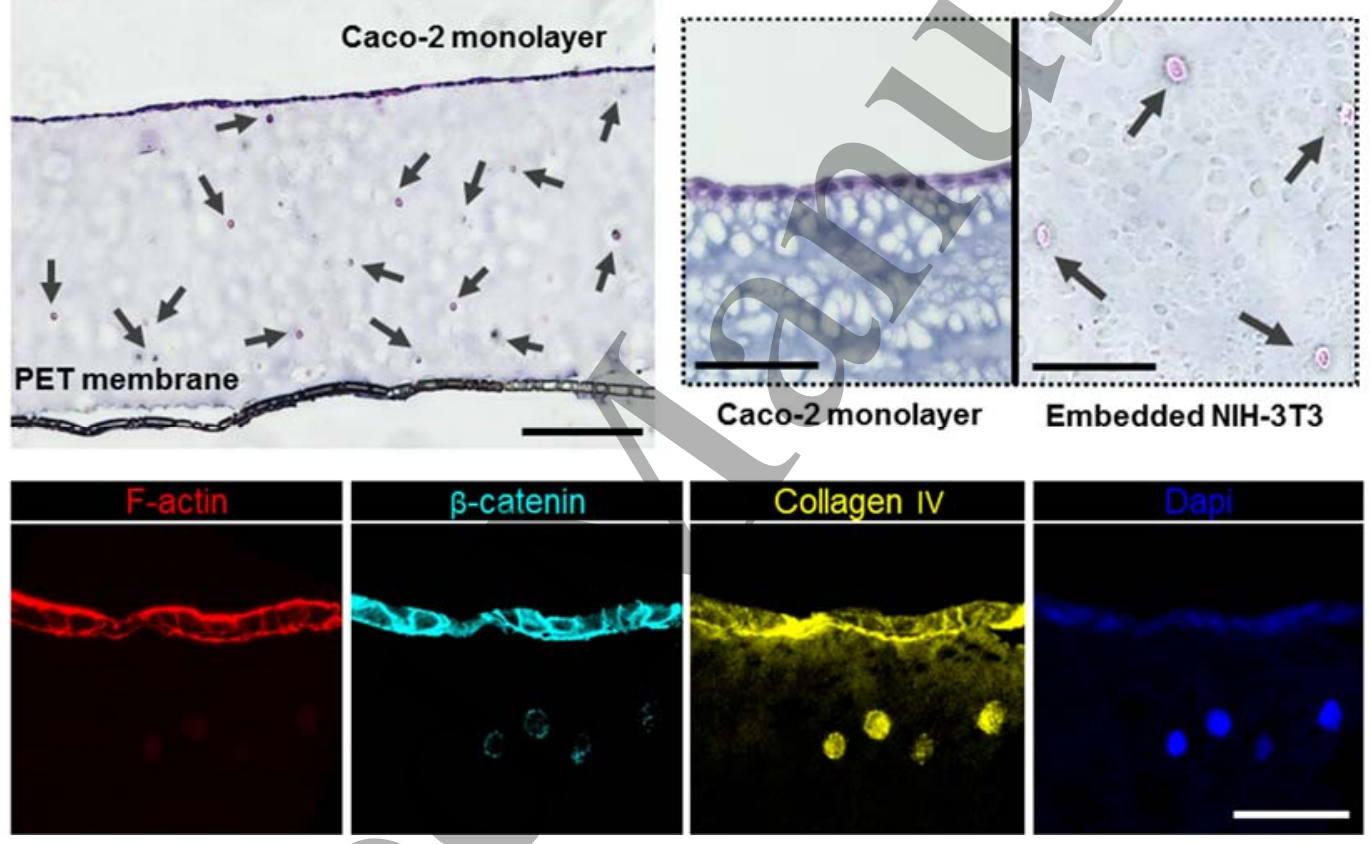

Embedded NIH-3T3

Figure 4. (a) Schematic to illustrate the NIH-3T3 fibroblast and Caco2 epithelial cell co-culture method in the hydrogel conetworks. (b) Cross-section of haematoxylin and eosin stained co-culture samples in hydrogel co-networks showing the formation of an intact epithelial monolayer at the top and a uniform distribution of the NIH-3T3 fibroblasts (arrows) throughout the hydrogel (left panel). The right panels shows detailed views of both cell types. Scale bars: $150 \mu \mathrm{m}$ (left) and $50 \mu \mathrm{m}$ (right). (c) Immunostainings for F-actin, $\beta$-catenin, and Collagen IV of a co-culture sample in hydrogel co-networks. Scale bar: $50 \mu \mathrm{m}$. DAPI was used to stain the nuclei. All samples were fixed and stained after 21 days of culture.

properties. In contrast, fibroblast-laden hydrogels without epithelial cells on top (controls) did not show any significant change from the basal TEER background values (Figure S6), which conclusively demonstrates that the increase in TEER was due to the formation of an epithelial barrier. TEER values of Caco-2 epithelial monolayers grown on GelMA-PEGDA hydrogels were significantly lower (up to 4-fold) than for cells grown on hard Transwell ${ }^{\circledR}$ inserts (Figure 5(c)). It should be noted that TEER values of Caco-2 cell monolayers grown on hard porous membranes increase already after 2-3 days in culture, while cells grown on GelMA-PEGDA hydrogels did not show an increase until 11-12 days of culture. This indicates a delay in epithelial layer formation when cells are grown on hydrogel substrates. It has been shown that the physical properties of cellular microenvironments play a crucial role in regulating cell division [44], collective cell 
migration [45], and, more importantly, in the maturing of tight junctions [46]. We therefore attribute the delay in epithelial monolayer formation and lower TEER values in the hydrogelcontaining samples to the soft mechanical properties of the
GelMA-PEGDA hydrogels (apparent elastic modulus of about $35 \mathrm{kPa}$ ) compared to the Transwell ${ }^{\circledR}$ inserts (about $2 \mathrm{GPa}$ ). This is also in agreement with previous findings for soft natural $[16,47]$ or synthetic hydrogels [26] which yield

a
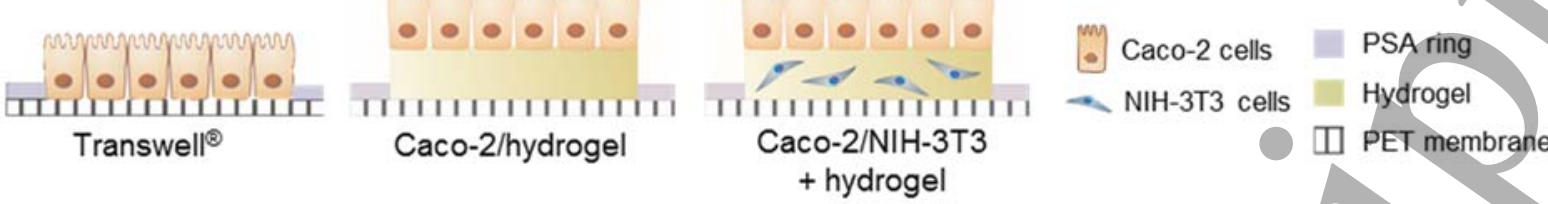

b

Day 8

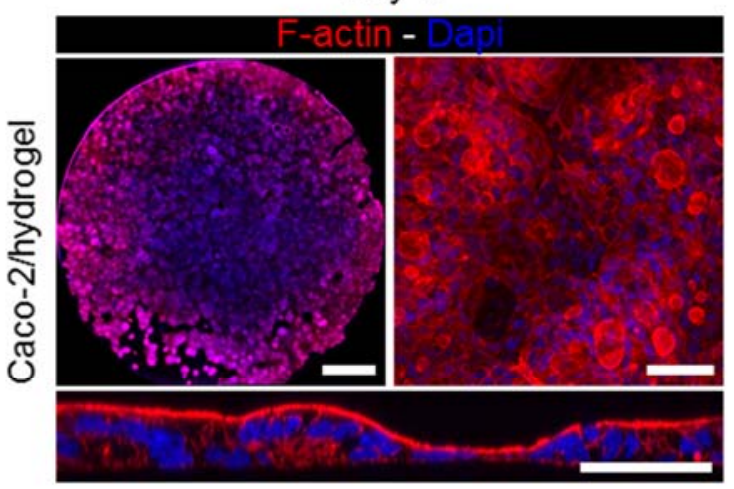

C

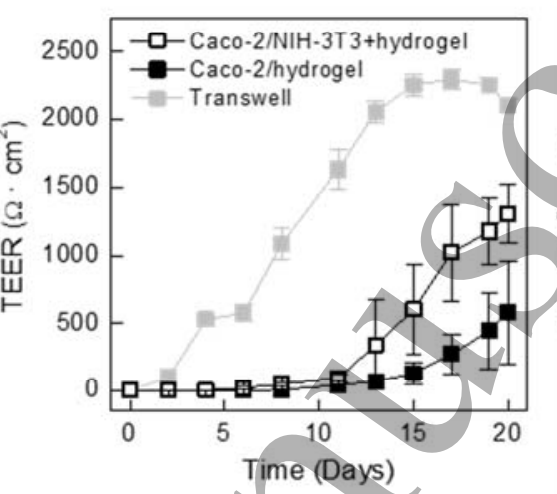

d

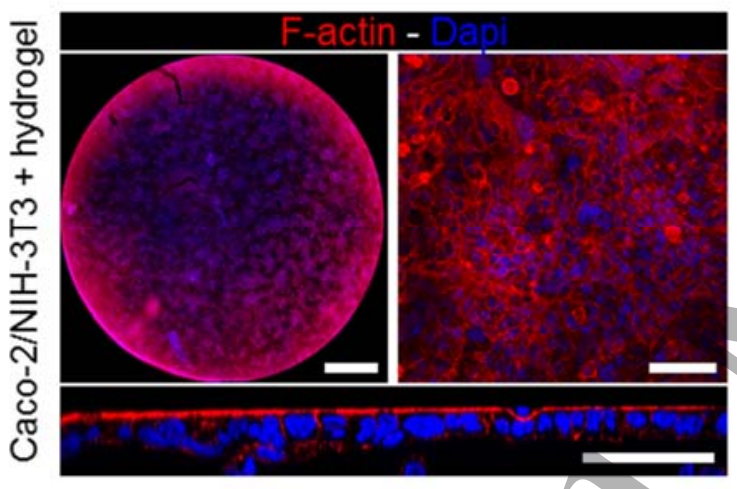

e
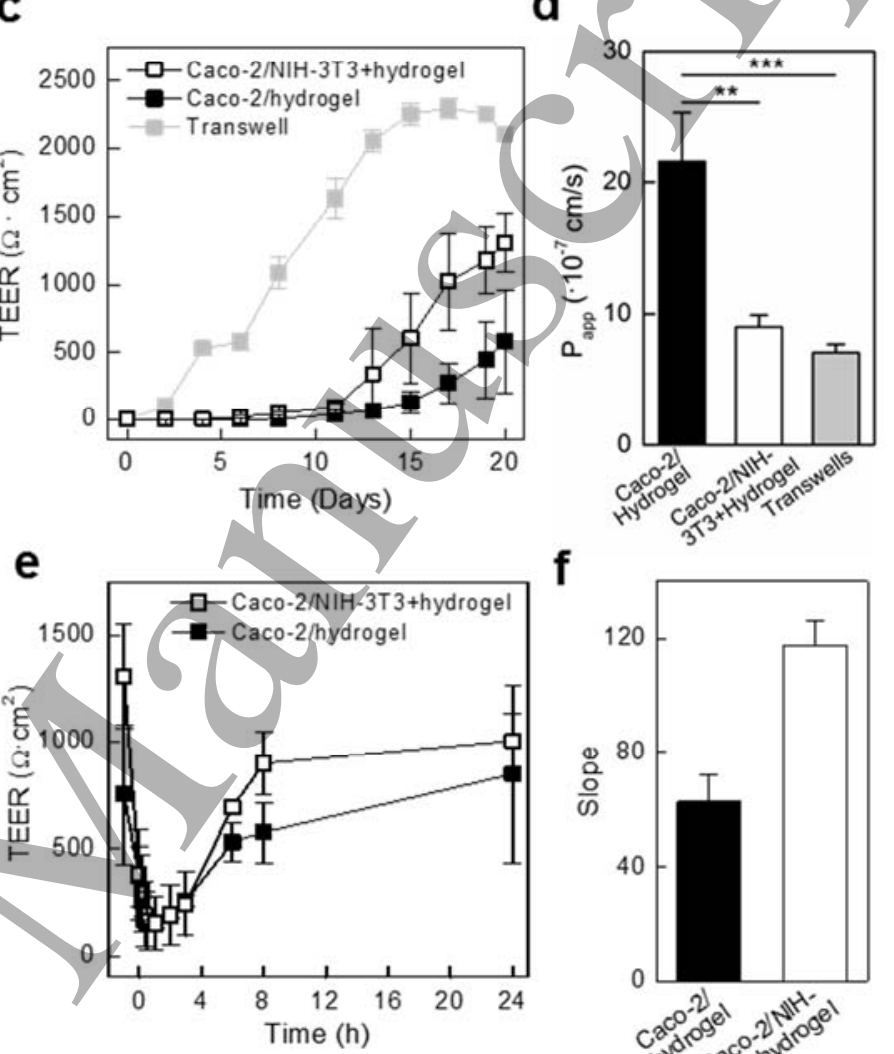

$f$

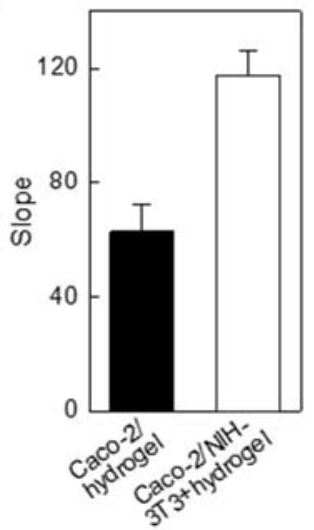

Figure 5. (a) Schematic illustration of the analysed cell culture configurations. (b) Immunostainings for F-actin and nuclei of Caco-2 cells grown on top of hydrogel co-networks without (top images) and with (bottom images) embedded NIH-3T3 cells after 8 days in culture. Left panels show tile-scan images of the entire hydrogel surface $\left(0.33 \mathrm{~cm}^{2}\right)(\mathrm{scale}$ bar $1 \mathrm{~mm})$, right panels are magnification images (scale bar $50 \mu \mathrm{m}$ ), and bottom panels are cross-sections views (scale bar $50 \mu \mathrm{m})$ of the corresponding samples. (c) Transepithelial electrical resistance (TEER) as function of cell culture time for epithelial monolayers grown on conventional Transwells ${ }^{\circledR}$ (grey) and on top of hydrogel co-networks with (white) and without (black) embedded NIH-3T3 fibroblasts. (d) Apparent permeability (Papp) of FITC-dextran $4 \mathrm{kDa}$ (FDA) through epithelial monolayers grown on conventional Transwell® (grey) and on top of hydrogel co-networks with (white) and without (black) embedded NIH-3T3 fibroblasts. Values are mean $\pm \mathrm{SD}(\mathrm{n}=3)$. (e) TEER evolution upon EDTA treatment of the epithelial monolayer grown on hydrogel co-networks in the presence or absence of embedded NIH-3T3. (f) Change of TEER over time from panel (e). Values are mean $\pm \operatorname{SD}(\mathrm{n}=3)$. $* * \mathrm{p}<0.005$ and $* * * \mathrm{p}<0.001$.

physiologically realistic vâlues while hard porous membranes typically result in unrealistically high TEER values [48]. Hydrogel-based materials thus present near in vivo-like mechanical properties providing cells with an appropriate environment to grow and form epithelial monolayers that closely resemble the in vivo tissue barrier properties. Also, the change in TEER over time (slope) differed considerably between fibroblast-laden or fibroblasts-free GelMA-PEGDA hydrogels. The presence of fibroblasts clearly accelerated the monolayer formation and the development of tight junctions, 
which led to TEER values having increased 2.5 -fold by day 21 (Figure 5(c)). To further characterize the barriers, we investigated their permeability using dextrans of different molecular weights as tracers for paracellular transport through tight junctions. Consistent with the lower TEER values, the epithelial barriers formed onto the GelMA-PEGDA hydrogels were more permeable to small $4 \mathrm{kDa}$ dextran than those formed on Transwells ${ }^{\circledR}$ inserts (Figure 5(d)). Moreover, among the hydrogel samples, those containing embedded fibroblasts showed less epithelial permeability than those without fibroblasts (Figure 5(d)). This permeability was sizeselective, as the transport of the large $70 \mathrm{kDa}$ dextran was significantly hindered (Figure S7). These results indicate that the presence of embedded fibroblasts in the hydrogel had a positive effect on the formation of a continuous and uniform epithelial monolayer with mature tight junctions that lead to higher TEER and lower permeability than in epithelial monolayer formed on hydrogels devoid of fibroblasts. As a biological relevant model drug, we evaluated the permeability of insulin across the fibroblast-laden GelMA-PEGDA hydrogel (Figure S8). The apparent permeability coefficent $\left(\mathrm{P}_{\text {app }}\right)$ obtained was $6 \pm 1 \times 10^{-8} \mathrm{~cm}^{-1}$, an order of magnitud higher than reported values for conventional Caco-2 monolayers on Transwell membranes [49,50]. This result was again consistent with the lower TEER values observed for the hydrogel-containing samples. Overall, these resutls showed that Caco-2 cells grown on fibroblast-laden GelMA-PEGDA hydrogels form a more physiological barrier, correlating better with the in vivo intestinal permeability and TEER values [51].

\subsection{Effects of stromal components on the recovery of the barrier function of the intestinal epithelium}

We used our in vitro model of the intestinal mucosa to mimic the loss of epithelial barrier integrity, a common occurrence under specific pathological conditions. Epithelial monolayers grown on fibroblast-laden or fibroblast-free GelMA-PEGDA hydrogels were grown for 21 days as previously described, and then treated shortly with the calcium-chelating agent EDTA to disrupt the tight junctions and increase permeability. Epithelial integrity was monitored by measuring TEER values at different time points from the moment we added the EDTA to 24 hours after removing it. As expected, treatment with EDTA reduced the TEER values in both samples to basal levels (Figure 5(e)). After removing EDTA, TEER values recovered due to the recovery of the tight junctions and associated barrier integrity of the epithelial monolayers. Interestingly, TEER recovered more quickly in epithelial monolayers grown on fibroblast-laden hydrogels than in those formed on fibroblast-free hydrogels (Figure 5(e, f)). These results indicate that the presence of stromal fibroblasts not only affects the Caco-2 monolayer growth rate, morphology, and permeability, but also the rate of recovery of a barrier following a temporary disruption of the tight junctions.

\section{Discussion}

Intestinal epithelial-stromal interactions play an important role in regulating fundamental biological processes both under physiological and pathological conditions $[9,21,52,53]$. It is therefore crucial to engineer more physiologically relevant in vitro models of intestinal mucosa that represent not only the epithelial but also the cellular and matrix components of the stroma. Despite recent advances, the set-ups are often highly complex which limits their use in routine assays [54,15]. Furthermore, most studies employed biodegradable hydrogels of only natural origins $[14,16]$, which greatly compromises the mechanical stability of the constructs over time and, thus, their life span.

Here we have presented a simple procedure to fabricate a conetwork formed by gelatine methacrylate (GelMA), a naturalderived polymer, and poly (ethylene glycol) diacrylate (PEGDA), a synthetic polymer, to produce a mechanically stable and long-lasting 3D model of the intestinal mucosa, which posses apparent elastic modulus values similar to those found in vivo $\approx 40 \mathrm{kPa})[55,56]$. The covalently crosslinked GelMA-PEGDA co-networks possess adjustable swelling, mesh size, degradábility, and mechanical properties, resulting in a versatile system that can emulate the physicochemical properties of tissue extracellular matrices, which combine rigid and soft networks to provide mechanical stability, celladhesion properties, and extracellular matrix remodelling capabilities [17,54]. We demonstrated that GelMA-PEGDA hydrogel co-networks can sustain viable and functional embedded fibroblasts that emulate the cellular component of the stromal compartment of the intestinal mucosa. In addition, we could show that epithelial cell monolayers can be successfully grown on top of the fibroblast-laden hydrogels. After 21 days in culture, epithelial monolayers formed on GelMA-PEGDA co-networks were nicely polarized and expressed typical epithelial markers. The effective tissue barrier function of the epithelial monolayers was evaluated by TEER and permeability measurements. We found that both were closer to physiological values reported for the intestine $\left(100-400 \Omega \mathrm{cm}^{2}\right)$ [57] when epithelial cells were grown on hydrogel co-networks than on standard Transwells ${ }^{\mathbb{\circledR}}$. . Using a model of pathological intestinal barrier disruption, we showed that the presence of fibroblasts in the artificial stroma accelerated the recovery of the tight junctions and the barrier function of the damaged epithelium returning them to physiological levels. Remarkably, the effects of the NIH-3T3 fibroblasts on the Caco-2 epithelial cells were noticeable despite using fibroblasts of a murine non-intestinal origin at low densities. NIH-3T3 fibroblasts have been extensively used in co-cultures with murine but also with human cells as feeder layers [58]. While it is well known that NIH-3T3 
favours cell growth through paracrine signalling, the underlying mechanisms are still not fully understood. Cocultures of fibroblasts and Caco-2 epithelial cells in conventional Transwell ${ }^{\circledR}$ inserts have been reported to enhance epithelial proliferation and differentiation via paracrine effects of the fibroblasts such as hepatocyte growth factor (HGF) [52] or keratinocyte growth factor (KGF) [59]. No tissue specificity for the fibroblast action was detected in these studies. In addition, mesenchymal-stromal cells have also been reported to accelerate tight junction assembly in epithelial monolayers [46]. Paracrine signalling also seems to be the main signalling source of NIH-3T3 fibroblasts in our mucosa model, as their growth appeared inhibited and we did not see any physical interaction between them and the epithelial cells.

Overall, our results demonstrate that GelMA-PEGDA hydrogel co-networks might be good candidates to produce long-lasting in vitro models of the intestinal mucosa with the epithelial and mesenchymal compartment being represented in a cell-spatial configuration recapitulating the one found in vivo. Combining natural and synthetic polymers to generate the scaffold we could ensure both the integrity of the construct for long periods of time (at least 21 days of culture) and the compatibility with cell growth and functionality of the embedded cells. Furthermore, we showed that embedded fibroblasts do express collagen IV, which indicates their capacity to remodel the surrounding matrix by creating new matrix or by degrading the existing one [16]. Thus, our PEGDA-GelMA hydrogel co-networks would allow to mimic in vivo interactions between mesenchymal and epithelial cells that would help us understanding better biological processes that depend on cell migration, cell-cell interactions and cellmatrix interactions, both under physiological and pathological conditions.

\section{Conclusion}

Our intestinal mucosa model based on GelMA-PEGDA hydrogel co-networks was able to mimic some of the features attributed to mesenchymal-epithelial interactions such as enhanced epithelial cell proliferation and barrier permeability, diffusivity properties that allow paracrine effects and accelerated tight junction recovery. As added benefits, our 3D model possesses a tissue-like architecture that includes both stromal and epithelial compartments arranged in a spatially relevant manner as well as in vivo-like mechanical properties of the substrate. This enables to model cell-to-cell and cell-tomatrix interactions with in vivo-like accuracy, which translates into more physiological values of intestinal permeability hallmarks (TEER and permeability). Our model provides a simplistic but still meaningful approach to obtain more physiologically relevant in vitro epithelial models at the cellular and functional levels. It can therefore be used to improve predictions of intestinal permeability in drug studies or to implement better epithelial disease models where an accurate reproduction of the interaction between different cell compartments is of crucial importance. These GelMAPEGDA co-networks should be able to accommodate additional relevant stromal cellular components such as the immune system (pivotal for regulating inflammatory responses, metabolic and cancerogenic processes) while being sufficiently versatile to mimic other simple or stratified epithelial tissues such as oesophagus, stomach, or skin.

\section{Acknowledgements}

Funding for this project was provided by a European Union Horizon 2020 ERC grant (agreement no. 647863 - COMIET), the CERCA Programme/Generalitat de Catalunya (2017SGR-1079), and the Spanish Ministry of Economy and Competitiveness (TEC2014-51940-C2-2-R, TEC201783716-C2-1-R, and the Severo Ochoa Programme for Centres of Excellence in R\&D 2016-2019). M. G.-D. would like to acknowledge financial support through the BEST Postdoctoral Programme, funded by the European Commission under the Horizon 2020 Marie SkłodowskaCurie Actions COFUND scheme (grant agreement no. 712754) and by the Severo Ochoa programme of the Spanish Ministry of Science and Competitiveness (Grant SEV-20140425, 2015-2019). The collaboration of the MicroFabSpace from IBEC is gratefully acknowledged. The results presented here only reflect the views of the authors; the European Commission is not responsible for any use that may be made of the information it contains.

\section{References}

[1] Hunyady B, Mezey E, Palkovits M 2000 Acta Physiol. Hung. 87 305-328

[2] Turner JR 2009 Nat. Rev. Immunol. 9 799-809

[3] Vancamelbeke M, Vermeire S 2017 Expert Rev. Gastroenterol. Hepatol. 11 821-834

[4] Artursson P, Karlsson J 1991 Biochem. Biophys. Res. Commun. 175 880-885

[5] Yanagawa F, Kaji H. Jang, Y-H, Bae H, Yanan D, Fukuda J, Qi H, Khademhosseini A 2011 J. Biomed. Mater. Res. A 97 93102

[6] Bischoff SC, Barbara G, Buurman W, Ockhuizen T, Schulzke J-D, Serino M, Tilg H, Watson A, Wells JM 2014 BMC Gastroenterol. 14 1-25

[7] Wang K, Wu L-Y, Dou C-Z, Guan X, Wu H-G, Liu H-R 2016 Gastroenterol. Res. Pract. 20169686238 1-6

[8] Maresca M, Pinton P, Ajandouz EH, Menard S, Ferrier L, Oswald IP 2018 Overview and Comparison of Intestinal Organotypic Model, Intestinal Cells, and Intestinal Explants Usedfor Toxicity Studies in Current topics in microbiology and immunology (Springer Nature Switzerland AG)

[9] Bosman FT, de Bruïne A, Flohil C, van der Wurff A, ten Kate J, Dinjens WW 1993 Int. J. Dev. Biol. 37 203-211

[10] Martínez E, St-Pierre JP, Variola F 2019 Adv. Phys. X 4 1-28 
[11] Torras N, García-Díaz M, Fernández-Majada V, Martínez E 2018 Front. Bioeng. Biotechnol. 6 1-7

[12] Duval K, Grover H, Han L-H, Mou Y, Pegoraro AF, Fredberg J, Chen Z 2017 Physiology (Bethesda) 32 266-277

[13] Turnbull IC, et al. 2014 FASEB J. 28 644-654

[14] Li N, Wang D, Sui Z, Qi X, Ji L, Wang X, Yang L 2013 Tissue Eng. Part C. Methods 19 708-719

[15] Matsusaki M, Hikimoto D, Nishiguchi A, Kadowaki K, Ohura K, Imai T, Akashi M 2015 Biochem. Biophys. Res. Commun. 457 363-369

[16] Pereira C, Araújo F, Barrias CC, Granja PL, Sarmento B 2015 Biomaterials 56 36-45

[17] Daniele MA, Adams AA, Naciri J, North SH, Ligler FS 2014 Biomaterials 35 1845-1856

[18] Fu Y, Xu K, Zheng X, Giacomin AJ, Mix AW, Kao WJ 2012 Biomaterials 33 48-58

[19] Aubin H, Nichol JW, Hutson CB, Bae H, Sieminski AL, Cropek DM, Akhyari P, Khademhosseini A 2010 Biomaterials 31 6941-6951

[20] Nichol JW, Koshy ST, Bae H, Hwang CM, Yamanlar S, Khademhosseini A 2010 Biomaterials 31 5536-5544

[21] Powell DW, Pinchuk IV, Saada JI, Chen X, Mifflin RC 2011 Annu. Rev. Physiol. 73 213-237

[22] Van Den Bulcke AI, Bogdanov B, De Rooze N, Schacht EH, Cornelissen M, Berghmans H 2000 Biomacromolecules 131 38

[23] Loessner D, Meinert C, Kaemmerer E, Martine LC, Yue K, Levett PA, Klein TJ, Melchels FPW, Khademhosseini A, Hutmacher DW 2016 Nat. Protoc. 11 727-746

[24] Li X, Chen S, Li J, Wang X, Zhang J, Kawazoe N, Chen G 2016 Polymers 8 1-15

[25] Habeeb AFSA 1966 Anal. Biochem. 14 328-336

[26] Castaño AG, García-Díaz M, Torras N, Altay G, Comelles J, Martínez E 2019 Biofabrication 11025007

[27] Flory PJ, Rehner J 1943 J. Chem. Phys. 11 512-520

[28] Peppas NA, Merrill EW 1977 J. Appl. Polym. Sci. 211763 1770

[29] Okeowo O and Dorgan JR 2006 Macromolecules 39 81938202

[30] Kontturi L-S, Collin EC, Murtomäki L, Pandit AS, Yliperttula M, Urtti A 2015 Eur. J. Pharm. Biopharm. 95 387-397

[31] Caballero D, Samitier J 2017 ACS Appl. Mater. Interfaces 9 4159-4170

[32] Benton JA, DeForest CA, Vivekanandan V, Anseth KS 2009 Tissue Eng. Part A 15 3221-3230

[33] Engler AJ, Sen S, Sweeney HL, Discher DE 2006 Cell 126 677-689

[34] Hönig W, Kula MR 1976 Anal. Biochem. 72 502-512

[35] Peppas NA, Hilt JZ, Khademhosseini A, Langer R 2006 Adv. Mater. 18 1345-1360

[36] Consultancy, T 2010 Fluorescein isothiocyanate dextran. Prod. Inf. 1-5

[37] Sigma 1997 Fluorescein Isothiocyanate-Dextran. Prod. Inf. 13

[38] Kaemmerer E, Melchels FP, Holzapfel BM, Meckel T, Hutmacher DW, Loessner D 2014 Acta Biomaterialia 10 25512562

[39] Swift J et al. 2013 Science 3411240104
[40] Cai Y, Xu C, Chen P, Hu J, Hu R, Huang M, Bi H 2014 J. Pharmacol. Toxicol. Methods 70 175-181

[41] Wells RG 2008 Hepatology 47 1394-1400

[42] Valls-Margarit, et al. 2019 Stem Cell Reports 13 1-14

[43] Rodriguez-Boulan E, Kreitzer G, Müsch A 2005 Nât. Rev. Mol. Cell Biol. 6 233-247

[44] Uroz M, Wistorf S, Serra-Picamal X, Conte V, Sales-Pardo M, Roca-Cusachs P, Guimerà R, Trepat X 2018 Nat. Cell Biol. 20 646-654

[45] Sunyer R, et al. 2016 Science, 353 1157-1161

[46] Rowart P, Erpicum P, Krzesinski J-M, Sebbagh M, Jouret F 2017 Stem Cells Int. 9717353

[47] Sung JH, Yu J, Luo D, Shuler ML, March JC 2011 Lab Chip 11 389-392

[48] Le Ferrec E, Chesne C, Artusson P, Brayden D, Fabre G, Gires P, Guillou F, Rousset M, Rubas W, Scarino ML 2001 Altern. Lab. Anim. 29 649-668

[49] Wood KM, Stone GM, Peppas NA 2010 Acta Biomater.6 4856

[50] Liu J, Werner U, Funke M, Besenius M, Saaby L, Fanø M, Mu H, Müllertz A 2019 Int JPharm. 560 377-384

[51] Gupta V, Doshi N, Mitragotri S. 2013 PLoS One.8 e57136e57136

[52] Göke M, Kanai M, Podolsky DK 1998 Am. J. Physiol. 274 G809-818

[53] Haffen K, Simon-Assmann P, Kedinger M, Grenier JF, Zweibaum Z 1981 C. R. Seances Acad. Sci. III. 293 807-812

[54] Wang H, Heilshorn SC 2015 Adv. Mater. 27 3717-3736

[55] Dou Y, Gregersen S, Zhao J, Zhuang F, Gregersen H 2001. Med Eng Phys 23 557-566

[56] Liao D, Yang J, Zhao J, Zeng Y, Vinter-Jensen L, Gregersen H 2003 Med Eng Phys 25:413-418

[57] Srinivasan B, Kolli AR, Esch MB, Abaci HE, Shuler ML, Hickman JJ 2015 J Lab Autom 20 107-126

[58] Hynds RE, Bonfanti P, Janes SM 2018 EMBO Mol. Med. 10 $139-150$

[59] Visco V, Bava FA, D'Alessandro F, Cavallini M, Ziparo V, Torrisi MR 2009 J. Cell. Physiol 220 204-213 